\title{
Gurugyam and Chuvthag Cemeteries in Ngari Prefecture, Tibet
}

\section{Institute of Archaeology, CASS and Cultural Relics Conservation Institute of Tibet Autonomous Region}

Keywords: Burials-Zhang-zhung (region); Chuvthag Cemetery and Gurugyam Cemetery (Ngari Prefecture, Tibet); Dzi beads; intercultural communication-China and Central Asia.

\section{Abstract}

From 2012 the 2014, the Institute of Archaeology, Chinese Academy of Social Sciences and other institutions conducted a series of excavations at the Gurugyam and Chuvthag Cemeteries in Ngari Prefecture, Tibet. The Gurugyam Cemetery is the largest and the most concentrated vertical shaft pit burial cemetery found to date in Ngari, and has close relationship with the Kardung (Kyunglung Silver Palace) City Site. Chuvthag Cemetery is the first archaeologically excavated cave tomb cemetery in Ngari Prefecture; the structures of the burials were completely preserved and these burials were rich in content, providing valuable information for the reconstruction of life in the ancient Zhang-zhung Kingdom period. The discovery and study on these two cemeteries are important for establishing the sequence of archaeological cultures in western Tibet, and they also contribute to research on the Zhang-zhung Civilization.

\section{A brief introduction to the cemeteries}

Gurugyam and Chuvthag Cemeteries are located on the upper reaches of the Langqên Zangbo River in Ngari Prefecture, Tibet; administratively, they belong to Monicêr Township in Gar County and Tholing Township in Zanda County at about $200 \mathrm{~km}$ distance from each other (Figure 1). As the cemeteries are so close to each other and their archaeological assemblages are largely identical, since 2012, the Institute of Archaeology, Chinese Academy of Social Sciences and Cultural Relics Conservation Institute of Tibet Autonomous Region have been doing joint research at these two cemeteries, conducting three years of archaeological excavation and achieving great scholarly results. The report of Gurugyam excavation in 2012 has already been published (Institute of Archaeology
2014), attracting much attention in the scholarly world. In 2013, further excavations at Gurugyam clarified the extent of the cemetery and its cultural characteristics; at the same time, the survey to the Chuvthag Cemetery and the reviewing and trimming of the fetched data were conducted. In 2014, at Chuvthag Cemetery a group of graves with hidden burial chambers were discovered and a large amount of important archaeological material were obtained.

\section{Gurugyam Cemetery}

Gurugyam Cemetery is located in Monicêr Township in Gar County close to the modern Bôn Gurugyam Monastery. It is located $15 \mathrm{~km}$ east of the town government at a longitude of $80^{\circ} 38^{\prime} 26^{\prime \prime} \mathrm{E}$, a latitude of $31^{\circ} 7^{\prime} 45^{\prime \prime} \mathrm{N}$, and an elevation of $4300 \mathrm{~m}$ asl. The cemetery is located on the first-level terrace on the north bank of the Langqên Zangbo River. In 2012, four graves were excavated, two of which being joint burials, the other two single interments, the joint burials containing poorly preserved human bones and the single interments having been deposited in on side flexed position. The grave goods encompass silk, gold masks, pottery wares, wooden objects, bronze items, iron objects, and a large number of animal bones. The brocade with bird-and-beast designs and the characters "wang hou 王侯 (kings and marquises)", the small gold masks and the large bronze objects indicate that the cemetery con- 
tained burials of some high-ranking individuals.

In 2013-2014, a total of seven new burials were excavated that were all located around those excavated in 2012. Below, we first discuss the main results of these two excavation campaigns.

1. Grave construction.

The cemetery has three cultural layers. Layer 1 consists of yellow topsoil and is $0.1-0.25 \mathrm{~m}$ thick. Layer 2 consists of yellow sandy soil and is $0.1-0.3 \mathrm{~m}$ thick. Layer 3 consists of brown soil and is $0.2-0.6 \mathrm{~m}$ thick. The graves are located below Layers 2 and 3, at a depth of $0.6-1.6 \mathrm{~m}$ below the present-day ground. The graves are mostly arranged in north-south direction and where the graves are more densely distributed they are located at 3-6m distance. Seven burials are all earthen pit stone chamber graves, four of them (2013M1-M3 and 2014M1) being located closely to the area excavated in 2012; they were larger, buried deeper, and more unified in form and content than the others, and belonged to the Tubo Kingdom period. The three others are located in the north part of the cemetery, about $60 \mathrm{~m}$ from the area excavated in 2012. They are smaller, shallower, and according to radiocarbon dating results likewise belong to the Tubo Kingdom period.

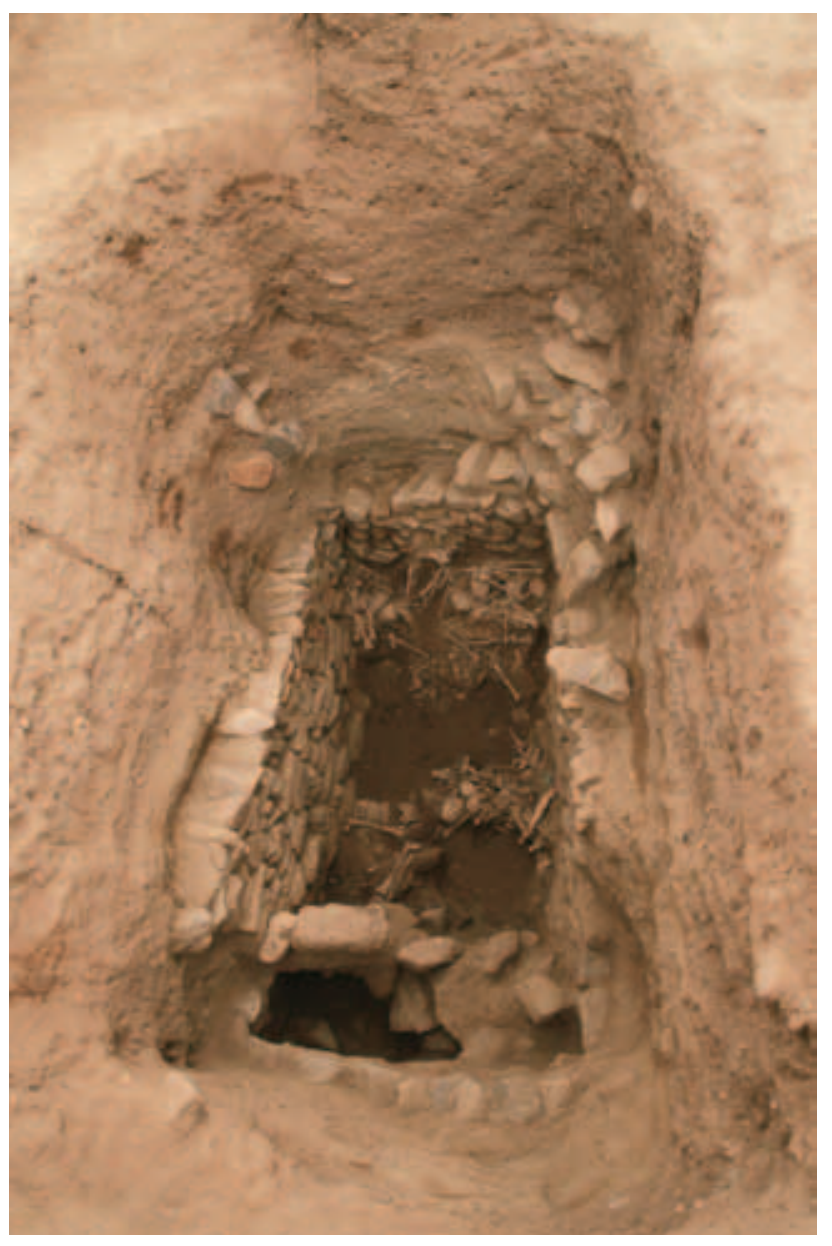

Figure 2 Gurugyam 2013M1 (W-E).
$2013 \mathrm{M} 1$ is located $8 \mathrm{~m}$ east of $2012 \mathrm{M} 1$. It is an earthenpit tomb located $0.5 \mathrm{~m}$ below the surface. It has a rectangular plan oriented at $285^{\circ}$, with a length of $6.6 \mathrm{~m}$, a width of $4.55 \mathrm{~m}$, and a depth of $4 \mathrm{~m}$. The upper layer of the grave opening forms a round sand pit with a medium diameter of $1.8 \mathrm{~m}$ and a depth of $0.3 \mathrm{~m}$ which contains large amounts of charcoal, bone and potsherds, a deposit that likely formed after the collapsing of the burial chamber. Below that feature lies the actual grave fill which is of grayish-brown color and rather soft and pure. At a depth of about $1 \mathrm{~m}$ in the grave filling there are sacrificial offering remains. The grave pit was partitioned into a western and an eastern compartment by stone slabs on this level. The western part was $3.2 \mathrm{~m}$ long and $2 \mathrm{~m}$ wide and contained human jaw bone fragments as well as bones from horse, sheep, dog, cattle, and other animals together with pottery wares, as well as fragments of bronze and iron objects, stones and the like. The eastern part did contain hardly any objects. At a depth of $3.95 \mathrm{~m}$ a rectangular stone chamber was found which measured $4 \times 2.2 \times 1.5 \mathrm{~m}$ with a wall $0.3-0.4 \mathrm{~m}$ thick. The top of the burial chamber had already collapsed and the stones were in disorder. The stone walls were coated in red pigment. Inside the chamber at a distance of $0.55 \mathrm{~m}$ from the western wall stones were piled up as a partition that was parallel to the western wall, providing the chamber with a head compartment. Part of the upper layer west of the stone partitioning had been removed, showing that after the chamber had been closed the grave was opened again from the western end and a new corpse was interred into the burial chamber, and then the wall was piled up again, constituting a clear case of secondary burial. Inside the chamber, 10 human skulls were found, eight of which placed next to the eastern wall of the chamber and two in its middle. The human skull bones were intermingled with human torso and limb bones as well as animal bones, none of the human skeletons being complete so that the burial positions could not be determined. Close to the eastern wall pottery ware fragments were found; in the middle close to the southern wall there were spouted pottery jars, iron bracelets, bronze bowl fragments, and glass beads. Under the pile of bones in the middle as well as under the grave goods there were large flat stone planks. Based on the placement, we can infer that the eastern bones and grave goods were placed into the grave during the first instance of burial and those in the middle followed during the second instance of burial (Figure 2).

$2013 \mathrm{M} 3$ is located $2.2 \mathrm{~m}$ north of $2013 \mathrm{M} 2$. It is an earthen pit grave with rectangular plan oriented at $269^{\circ}$ and measuring $3.1 \times 2.9 \times 3.4 \mathrm{~m}$. The grave fill is of grayish-brown color and loose in consistency; it contains a small number of animal bone fragments. At a depth of $2.5 \mathrm{~m}$ a rectangular chamber made of stones came to light which measured $1.65 \times 1.2 \times 1.3 \mathrm{~m}$ with a wall thickness of $0.3 \mathrm{~m}$. The top already collapsed and the stone lining had fallen into the burial chamber. The stone walls were painted in red color. The tomb occupant was buried in flexed position and was placed in the western side of the 
chamber with his head facing north and face toward the east; close-by there was a ram's head and in the eastern part of the chamber there were four more ram's heads as well as cattle bones and iron fragments (Figure 3).

Burial 2014M1 is located on the southern side of the cemetery, $60 \mathrm{~m}$ south of 2013M2. It is a rectangular earthen pit grave oriented at $205^{\circ}$. The grave fill is grayish-brown in color and has a loose texture; it contains horse bones and a considerable number of stone piles. Because of the collapse of the chamber, there are some holes in between the piled-up stones, allowing for sandy silt to leak in. On the western side, there is a round pit of $2 \mathrm{~m}$ diameter and $2.8 \mathrm{~m}$ depth, and the wall has a $0.2 \mathrm{~m}$ thick layer of silt. At a depth of $0.3 \mathrm{~m}$ within the grave fill stone piles were found, among which one in the southeastern and one in the southwestern corners are probably remains of sacrificial activities. In the southwestern corner a pile of animal bones and pottery as well as iron fragments and a large quantity of charcoal were piled up in several layers building a step-like structure; the pile in the southeastern corner was more chaotic, consisting mainly of wooden fragments stacked into an unidentifiable form. The top of the burial chamber was $2.6 \mathrm{~m}$ below the opening of the grave pit, stone boulders and stone slabs were piled up in a chaotic manner, and the stone slabs being close to the four grave walls, on them lying large piles of stone

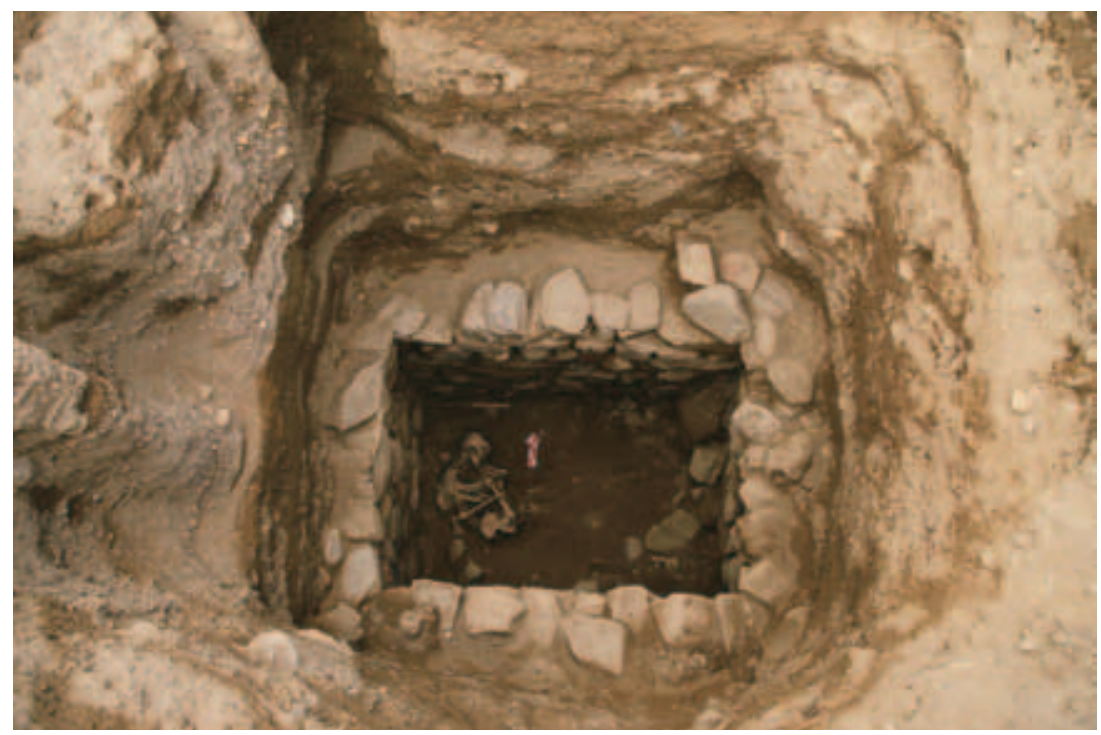

Figure 3 Gurugyam 2013M3 (S-N).

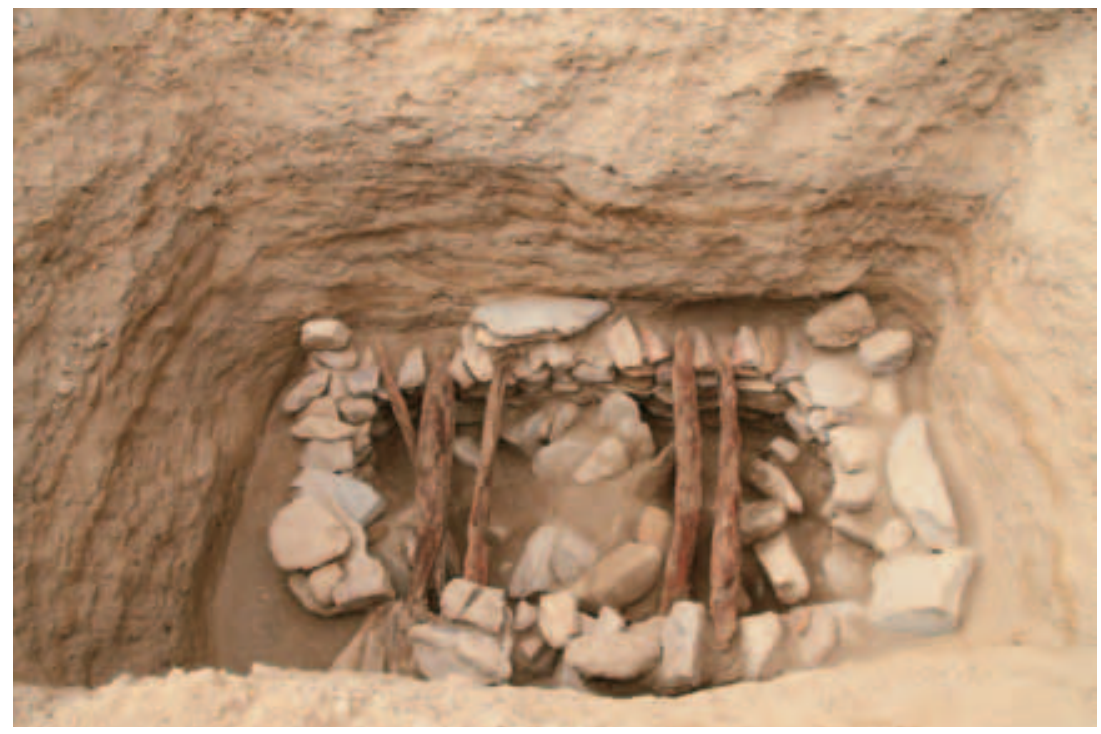

Figure 4 Gurugyam 2014M1 (top, NW-SE). boulders. Below the stones making

up the grave cover there were eight transverse wooden beams, only four of them remaining in place on each side, the others having been crushed by the cover stones, many of them having fallen into the chamber itself and having come to lie stacked up in chaotic piles (Figure 4). Among the stone boulders making up the grave cover there were iron cutters, iron belt buckles, and a small number of animal bones. The stone chamber was rectangular in plan, measuring $2.3 \times 1.3 \times 1.4 \mathrm{~m}$ with a thickness of $0.3 \mathrm{~m}$. On the bottom of the chamber in the western part there were traces of a wooden coffin with human skull and limb bones and a small number of sheep bones next to them, all randomly scattered. Beneath the wooden coffin there were stone slabs possibly building a stone coffin platform. In the middle and on the eastern side of the burial chamber human skull and limb bones were found, but apart from a single iron arrowhead and one potsherd no further objects were found (Figure 5).

2. Unearthed artifacts.

In the newly discovered seven burials the number of grave goods was limited, most of them being pottery wares, iron objects, and a small number of glass beads.

One spouted pottery jar (2013M1:1): red fine clay ware with a pointed lip, outward-flaring rim, high neck, globular body, and a small flat foot with a slightly concaved middle. On one side of the vessel body there is a handle decorated with four $\mathrm{W}$-shaped wave patterns; on the other side there is a tubular-shaped spout, which is connected to the neck by a band-shaped handle which had impressed patterns of circles and lines. On shoulders and neck of the jar there is a row of bowstring pattern, and on the vessel body there are three rows of incised vertical line pattern. 
The rim diameter is $7.2 \mathrm{~cm}$, the bottom diameter is $5 \mathrm{~cm}$, and the vessel is $9.5 \mathrm{~cm}$ high (Figure 6).

Two double-handled jars: red coarse sandy ware with an angular lip, straight mouth, two handles, a globular body, and a round bottom. The body below the neck is decorated with cord mark pattern. The object is covered with a thick layer of soot. 2013M2:3 has a rim diameter of $24 \mathrm{~cm}$, a belly diameter of 27 , and a remaining height of $16 \mathrm{~cm}$.

One pottery bowl (2013M2:2): red coarse sandy ware with a square lip, a flaring mouth, a slanting body, and a round bottom. On vessel surface and bottom there are impressed vertical cord marks. The vessel has a rim diameter of $12.3 \mathrm{~cm}$, a bottom diameter of $7 \mathrm{~cm}$, and a height of

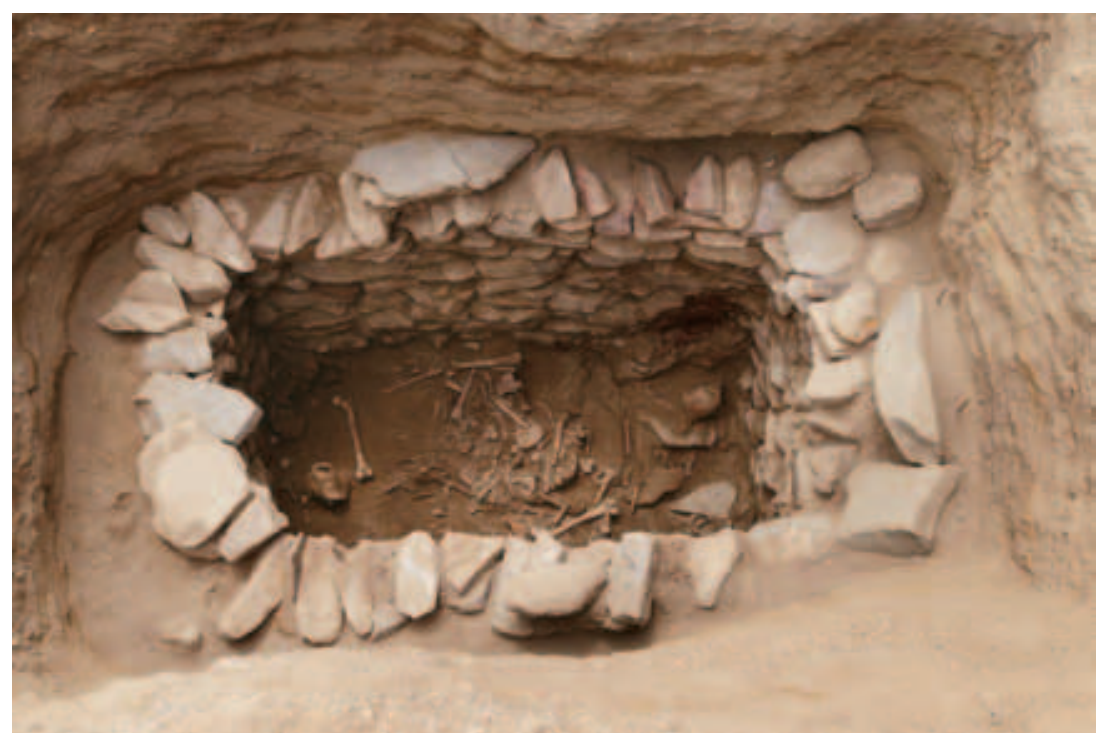

Figure 5 Gurugyam 2014M1 (chamber, NW-SE).

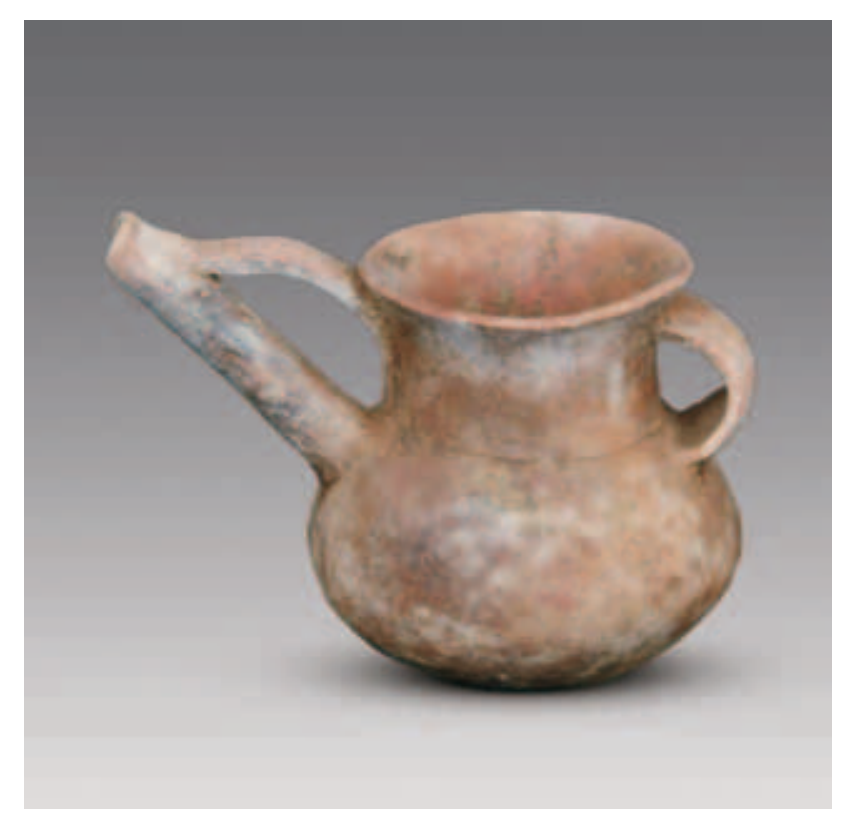

Figure 6 Spouted pottery jar (Gurugyam 2013M1:1).
$5 \mathrm{~cm}$.

Two high-stemmed cups: 2013M2:4 is a red coarse sandy ware with black slip. The vessel has a square lip, straight mouth, slightly globular body, and high ring foot. On each side there is a triangular lug. Below the belly and above the foot there is a row of vertical cord mark pattern. The rim diameter is $6.5 \mathrm{~cm}$, the foot diameter is $4 \mathrm{~cm}$, and the vessel is $9.5 \mathrm{~cm}$ high (Figure 7).

One pottery cup (2013M2:5): this vessel has a rounded lip, outward-flaring mouth, slanted body, and small flat foot. The rim diameter is $7 \mathrm{~cm}$, the remaining height measures $5.6 \mathrm{~cm}$.

One iron sword (2013M2:7): the piece is severely corroded. The blade is flat and has an I-shaped hand guard. It has a remaining length of $50 \mathrm{~cm}$, a width of 4.2 , a hilt length of $13 \mathrm{~cm}$, and a thickness of $0.5 \mathrm{~cm}$ (Figure 8 ).

One iron belt buckle (2013M2:8): the piece is severely corroded. It has a round ring shape with a tongue in the middle. The diameter is $4 \mathrm{~cm}$ and the thickness is $1 \mathrm{~cm}$.

Two iron horse bits: the objects are severely corroded. They come in two types. 2013M2:10 has a remaining length of $9.6 \mathrm{~cm}$ and a diameter of $1.5 \mathrm{~cm}$.

Three iron arrowheads: 2013M2:17 is a double-winged arrowhead with a tang, a length of $9.5 \mathrm{~cm}$, a maximum width of $2 \mathrm{~cm}$, and a thickness of $0.5 \mathrm{~cm}$.

One iron basin (2013M2:15): the object is badly fragmented and rusty. It is round with a wide opening,

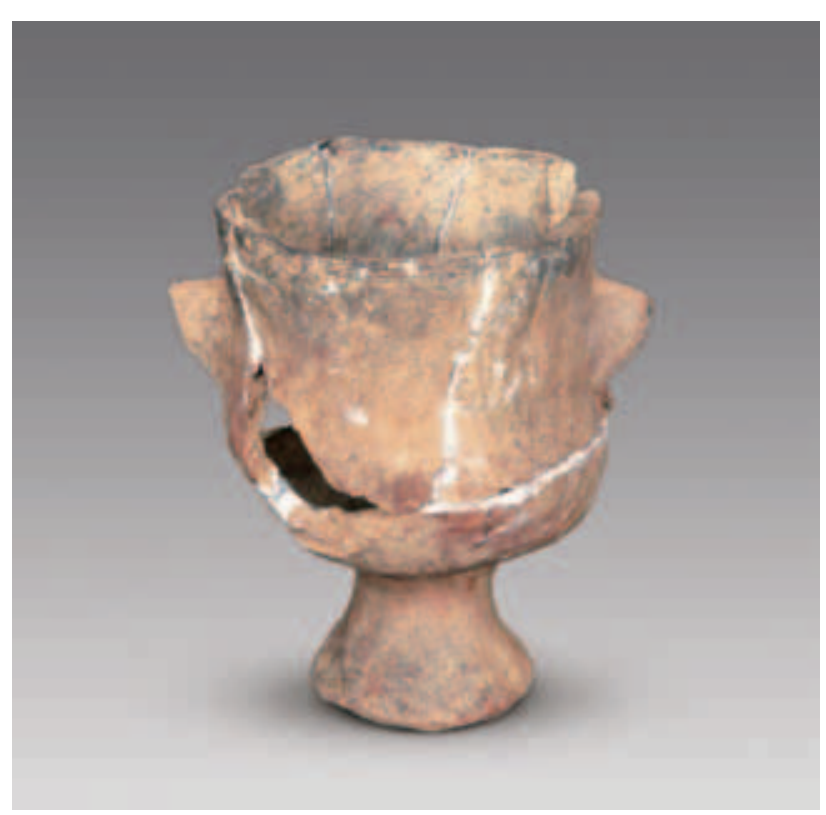

Figure 7 High-stemmed pottery cup (Gurugyam 2013M2:4). 
slanted sides, and a flat bottom. The rim diameter is $40 \mathrm{~cm}$ and the height is $3.3 \mathrm{~cm}$.

One bronze spoon handle (2013M2:9): this object is narrow and thin with a rectangular cross section. The handle is $19.5 \mathrm{~cm}$ long, $0.5 \mathrm{~cm}$ wide, and $0.3 \mathrm{~cm}$ thick.

One bronze-mounted wooden bowl (2013M1:4): this object is fragmented. It is a wooden bowl with the rim mounted with bronze and secured with rivets. The diameter is $18 \mathrm{~cm}$ and the height is $5 \mathrm{~cm}$.

One gold-plated silver sheet (2013M2:14): this item was broken into several dozens of pieces and the original form is unclear. The largest piece measured $3 \mathrm{~cm}$ in size. The whole surface is gilt and the back is blackened and showing wooden fragments, so there was likely originally a wooden base to it. The original object was perforated along the rim with holes of a diameter of $0.15 \mathrm{~cm}$. The original length was probably $12 \mathrm{~cm}$ and the width $10 \mathrm{~cm}$ (Figure 9).

A pair of gilt bronze earrings (2013M2:1): both objects are similarly ring-shaped. One of them has a gap on top, a small round ring on the outside close by, and an object of unclear shape inside. The other one consists of a completely closed circle with a notch closing it. In diameter it measures $1.4 \mathrm{~cm}$ and it is $0.1 \mathrm{~cm}$ thick.

One talc ring (2013M2:12): the object is milky-white in color. It has a diameter of $2.2 \mathrm{~cm}$ and is $0.3 \mathrm{~cm}$ thick (Figure 10:right).

Two crescent-shaped talc ornaments. 2013M2:13-1 is of milky-white color. It is $7 \mathrm{~cm}$ long with a maximum width of $0.6 \mathrm{~cm}$ (Figure 10:left top). 2013 M2:13-2 is of milky-white color. It is $8 \mathrm{~cm}$ long with a maximum width of $0.8 \mathrm{~cm}$ (Figure 10:left bottom).

One horn object (2013M2:6): the object is made of an ox horn and the ends are indented into a $\mathrm{V}$ shape. The object is $16 \mathrm{~cm}$ long with a maximum diameter of $3 \mathrm{~cm}$ and an indentation of $4.5 \mathrm{~cm}$ depth (Figure 11).

Three glass beads: they are globular and perforated. The surface is blue and the inside is yellow. 2012M2:181 has an outer diameter of $0.6 \mathrm{~cm}$ and a hole diameter of $0.1 \mathrm{~cm}$.

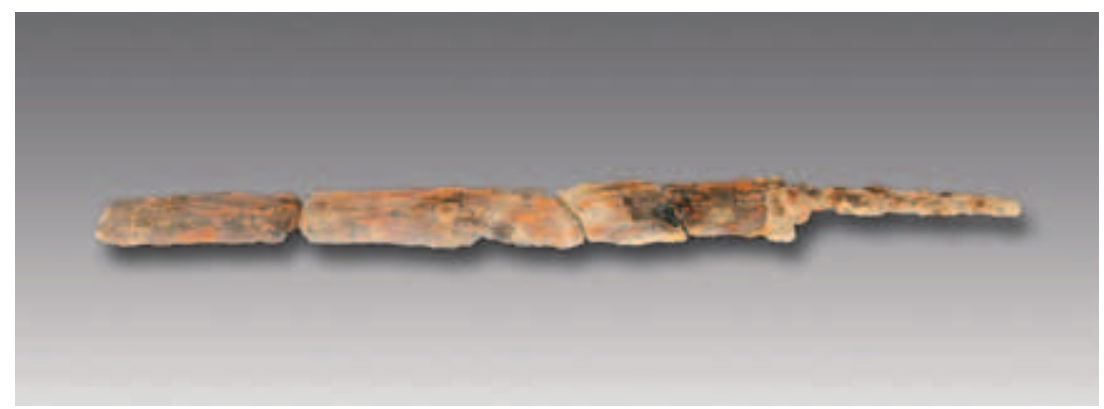

Figure 8 Iron sword (Gurugyam 2013M2:7).

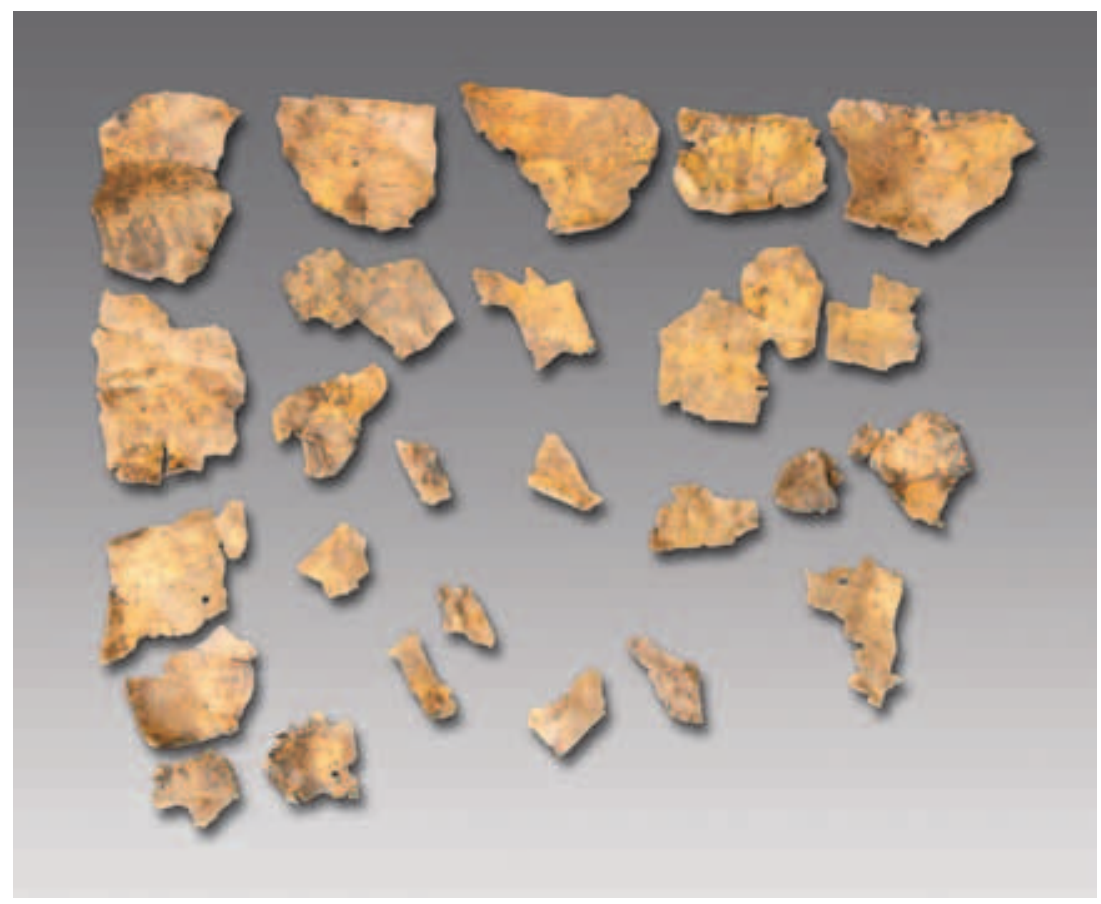

Figure 9 Fragments of gilt silver ornaments (Gurugyam 2013M2:14).

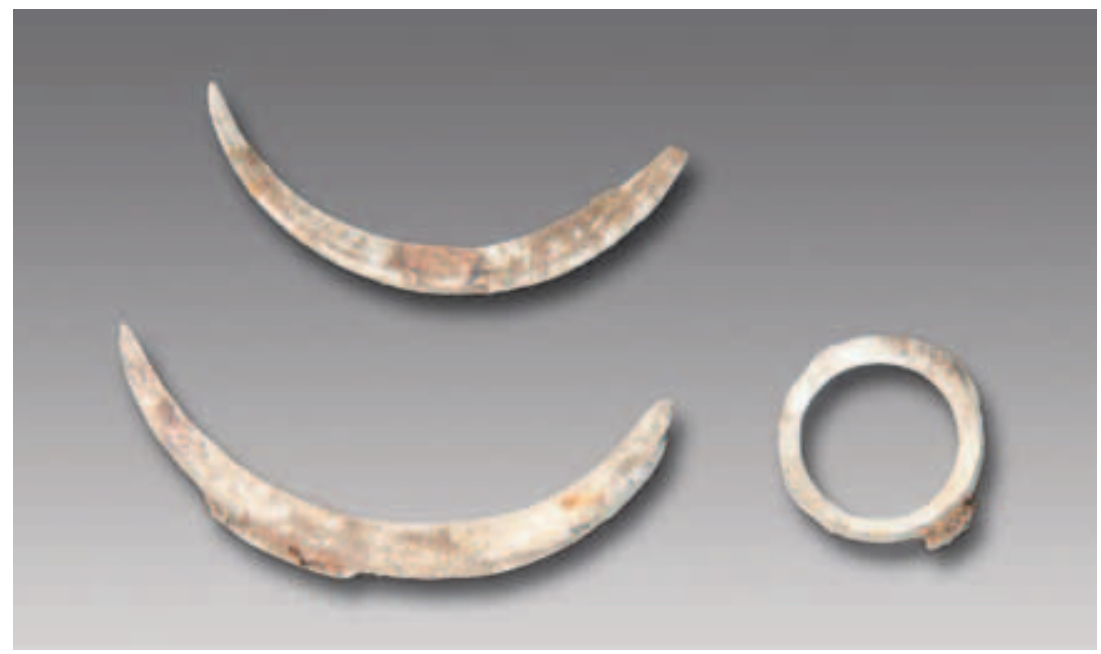

Figure 10 Talc objects.

Left top. Crescent-shaped ornament (Gurugyam 2013M2:13-1); left bottom. Crescent-shaped ornament (Gurugyam 2013M2:13-2); right. Ring (Gurugyam 2013M2:12). 


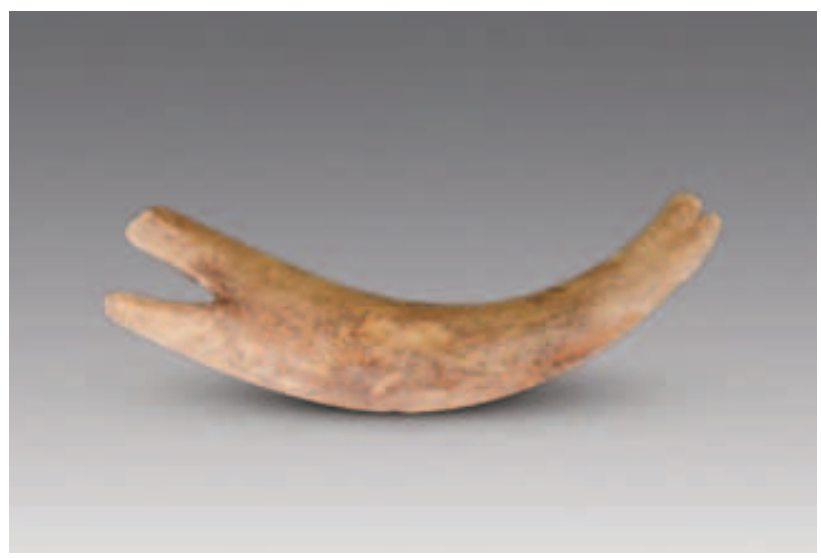

Figure 11 Horn object (Gurugyam 2013M2:6).

\section{Chuvthag Cemetery}

Chuvthag Cemetery is located $0.5-1.5 \mathrm{~km}$ west of Tholing Town, the Zanda County seat. The geographic coordinates are longitude $79^{\circ} 47^{\prime} \mathrm{E}$, latitude $31^{\circ} 29^{\prime} \mathrm{N}$, and an elevation of $3710 \mathrm{~m}$ asl. According to survey and excavation work, this cemetery can be divided into three zones arranged into triangular shape with a distance of $1 \mathrm{~km}$ between each other. Zone I is located on the hillside west of Chuvthag Gully and here two cave burials have been found; Zone II is located on the second-level terrace on the southern bank of the Langqên Zangbo River; here, six cave burials have been discovered. Zone III is located outside Zanda Middle School; because the villagers quarry earth for brick production at this location, this part of the site has been largely destroyed and was not excavated further (Figure 12).

1. Grave construction.

1.1 Burials in Zone I:

During the reconstruction of Par-Zanda Highway, two burials were discovered (2010M1 and 2010M2); the passages and part of the chambers were severely disturbed, but from the remainders it is still possible to restore the construction of the cave burials and their passages.

The burial chamber of M1 was roughly square, sealed with stone boulders, had a flat roof and is $2.9 \mathrm{~m}$ wide, $2.8 \mathrm{~m}$ deep, and $1.9 \mathrm{~m}$ high. On the right side of the chamber there was a wooden coffin of $1.7 \mathrm{~m}$ long, $1.5 \mathrm{~m}$ wide and $0.8 \mathrm{~m}$ high. The tomb occupant was interred in on side flexed position. Beneath the wooden coffin there was a small pottery vessel, to the east of the coffin there was a large number bones of cattle, sheep/goat, horse, and other animals. The grave goods include a gold mask, silk items, a large number of pottery wares, iron swords, iron arrowheads, iron horse bits, square wooden trays, basketry items, stone boxes, and stone rods.

M2 is located $50 \mathrm{~m}$ north of M1 and it was similar in form to M1 but severely disturbed. The passage was oriented at $45^{\circ}$ and the entrance was sealed with stone boulders. The burial chamber was in square plan, being $1.4 \mathrm{~m}$ long with an extant height of $1.5 \mathrm{~m}$. A rectangular wooden coffin was located in the right side of the chamber; the coffin measured $0.61 \times 0.55 \times 0.8 \mathrm{~m}$, and the grave goods were placed both inside and outside the coffin on its left site including also cattle and sheep bones. The grave goods encompass pottery jars, wooden weaving tools, wooden spindle whorls, bronze basins, bronze bracelets, square wooden trays, round lacquer plate, small wooden buckets, color-painted wooden boxes, wooden fire-making tools, and basketry items .

\subsection{Burials in Zone II.}

The stratigraphy of this zone can be divided into two cultural layers. Layer 1 consisted of a gravel and sandy soil accumulation of a thickness of $0.7-2.5 \mathrm{~m}$, material that was washed downhill from the top of the mountain slope. Layer 2 consisted of yellow sandy soil that is the primary deposit. The grave opening lies below Layer 1, intruding into the sandy layer. In this zone, six burials have been excavated, one of them (2013M1) having been excavated in the manner of a rescue excavation while the other five (2014M1 through M5) were regularly excavated. All of them were cave burials with passages (Figure 13).

2014M1 is located in the northern part of the cemetery at about $9 \mathrm{~m}$ distance from the cliff. It consisted of a vertical passage and a burial chamber. The passage opening was in irregular rectangular plan and oriented at $225^{\circ}$ with a length of $1.9 \mathrm{~m}$, a maximum width of $0.8 \mathrm{~m}$, and a depth of $3.3 \mathrm{~m}$. The western end was destroyed by a later ditch. The passage was filled with pebbles and sandy soil that were densely packed. The wall on the southern side

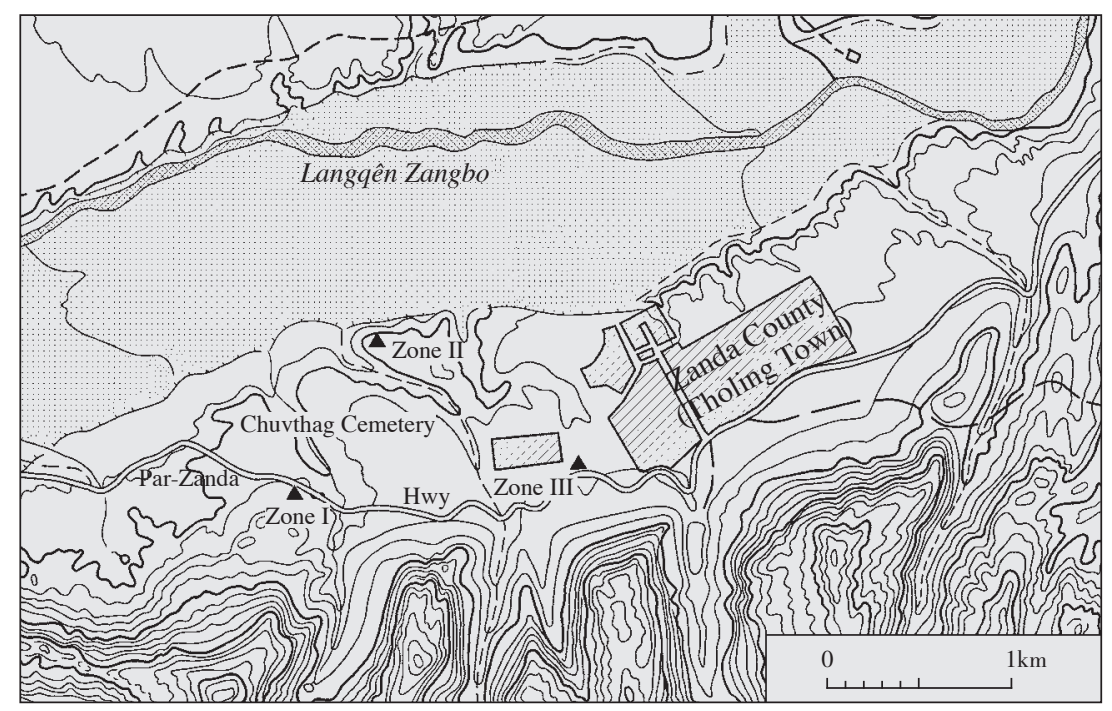

Figure 12 Layout of Chuvthag Cemetery. 
was irregular and slightly concave; of the wall on the southern side, three hollows remained. On the bottom of the passage, the burial chamber was opened on the eastern side toward the mountain side. The opening of the burial chamber was arched, being $0.6 \mathrm{~m}$ wide and $1 \mathrm{~m}$ high (Figure 14). The plan of the chamber was irregular with a width of $1.5 \mathrm{~m}$, a depth of $1.6 \mathrm{~m}$, and a height of $1.3 \mathrm{~m}$. The roof was mostly flat, the bottom was about $0.3 \mathrm{~m}$ lower than the bottom of the passage. There was a $1 \mathrm{~m}$ high accumulation of sandy soil inside the burial chamber and it was clear that water had come in, leaving the bones and grave goods in disorder. The deposit in the burial chamber consisted of three layers. The top layer contained severely fragmented human bones and potsherds, all of which were located in the left part of the chamber. The middle layer contained larger human bones, a small number of large potsherds, and hip and leg bones, all of them located on the left side of the opening of the chamber. The lower jaw bone was located in the middle of the left wall of the chamber, and between the two vertebrae and rib bones were found. On the right side of the chamber there were also a few small fragments of limb bones. The human bone fragments found on the left side of the burial chamber in the lower layer were rather chaotic and broken into tiny fragments. On the right side of the chamber there were three small pottery jars (Figure 15). No burial receptacles were found inside the burial chamber.

$2014 \mathrm{M} 2$ was located next to $2014 \mathrm{M} 1$, about $1.2 \mathrm{~m}$ south of it. The opening of the passage was even and rectangular, oriented at $225^{\circ}$, with a length of $1.96 \mathrm{~m}$, a width of $0.5 \mathrm{~m}$, and a depth of $5.1 \mathrm{~m}$. Close to the opening the walls on both sides showed deep indentations left by ropes. The upper part of the passage was filled with pebbles and sandy soil that was rather loose. At a depth of $2.6 \mathrm{~m}$ from the opening of the passage, on both the left and the right side there was a second-level ledge cut into the grave wall that was covered with stone slabs with pebble stones filling the gaps.

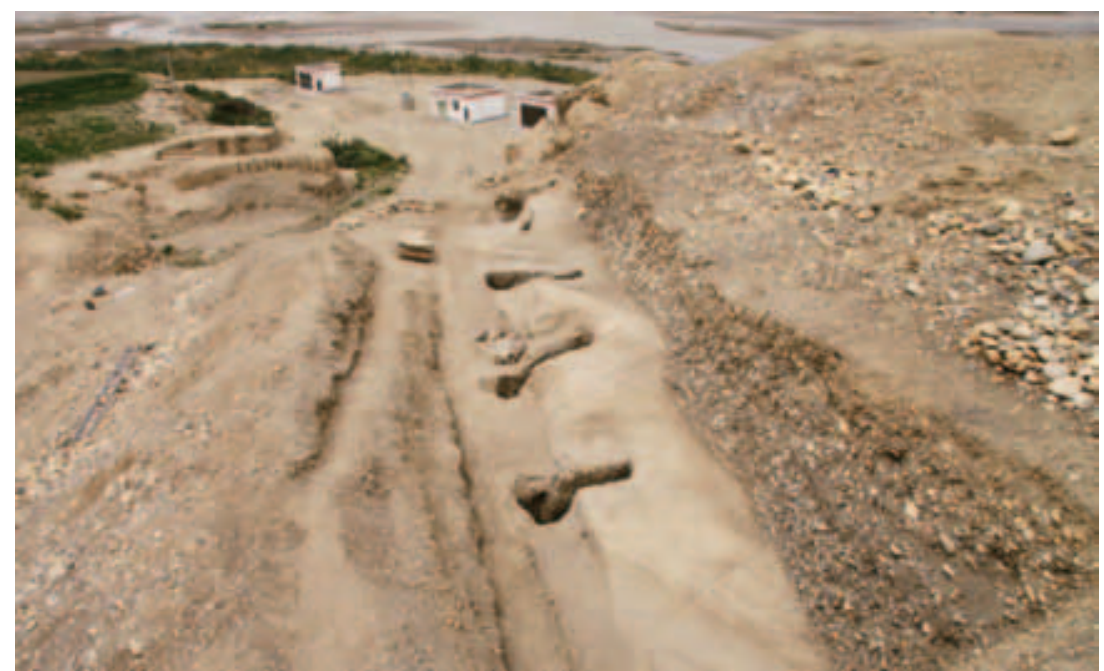

Figure 13 The area of Chuvthag Cemetery excavat ed in 2014 (SE-NW).

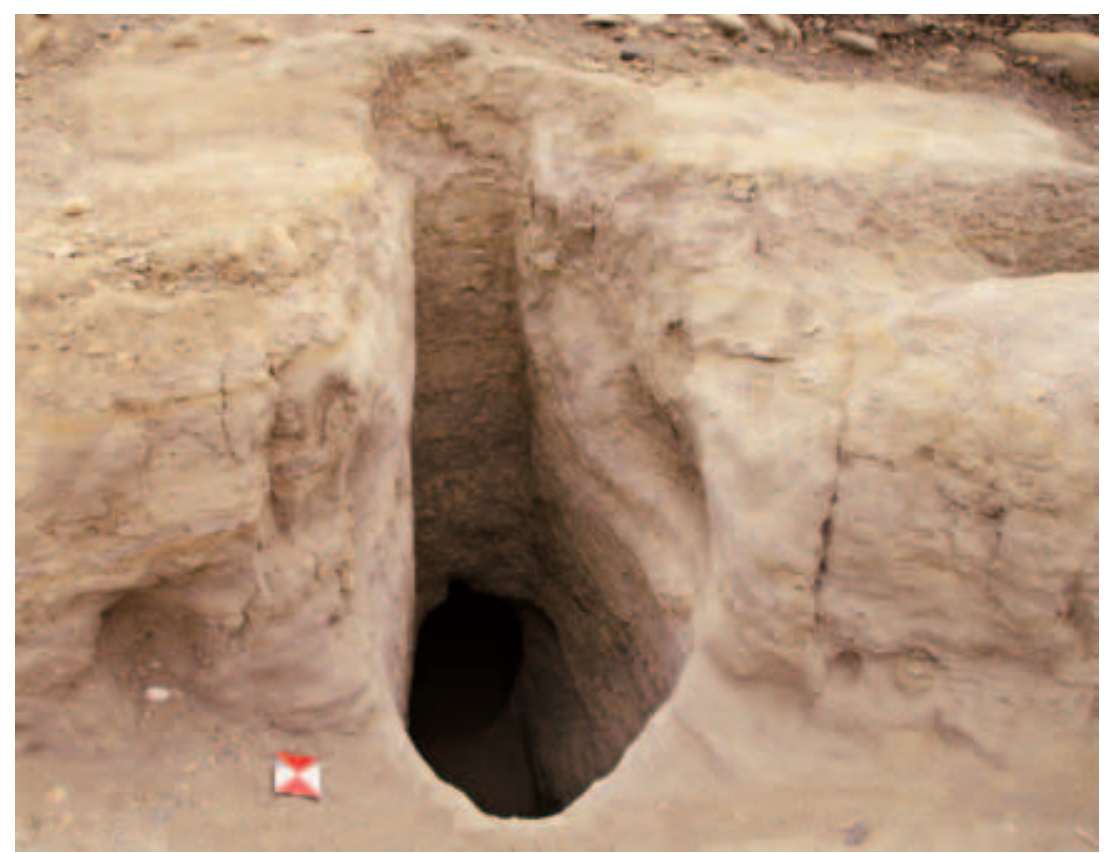

Figure 14 Chuvthag 2014M1 (SW-NE).

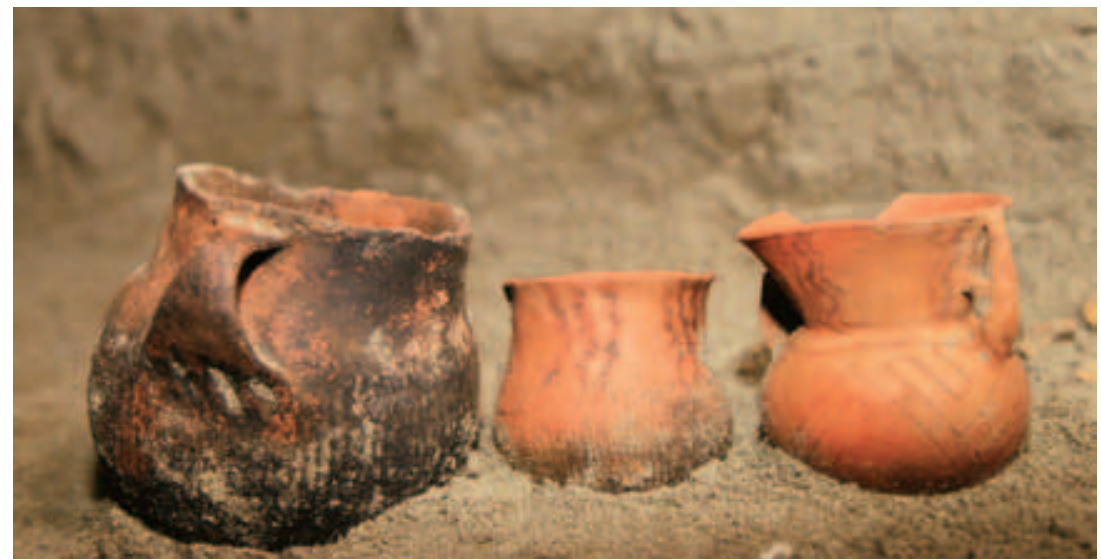

Figure 15 Pottery jars on the right wall of the chamber of Chuvthag 2014M1 (NW-SE). 
Below the stone slabs the passage was hollow that did not contain any soil. Both walls of the passage were filled with small indentations, six or seven on each of them, which had diameters of $15-20 \mathrm{~cm}$. The burial chamber was opened on the eastern side at the bottom of passage. The chamber opening was arched with a width of $0.7 \mathrm{~m}$ and a height of $1 \mathrm{~m}$. The chamber was rectangular in plan with a width of $3.1 \mathrm{~m}$, a depth of $2.6 \mathrm{~m}$, and a height of $1.7 \mathrm{~m}$. In the middle of the chamber there was a protrusion, dividing it into a left and a right part (Figure 16). There were four small recesses in the walls of the burial chamber that contained basketry items. In the middle of the left side there was a wooden coffin containing the bones of the burial occupant. The wooden coffin was rectangular, measuring $1.1 \times 0.62 \times 0.75 \mathrm{~m}$. There was no lid; only textiles covered the coffin opening. In the upper layer there was a small amount of debris consisting of bronze beads and other ornaments sewn onto textiles. Be-

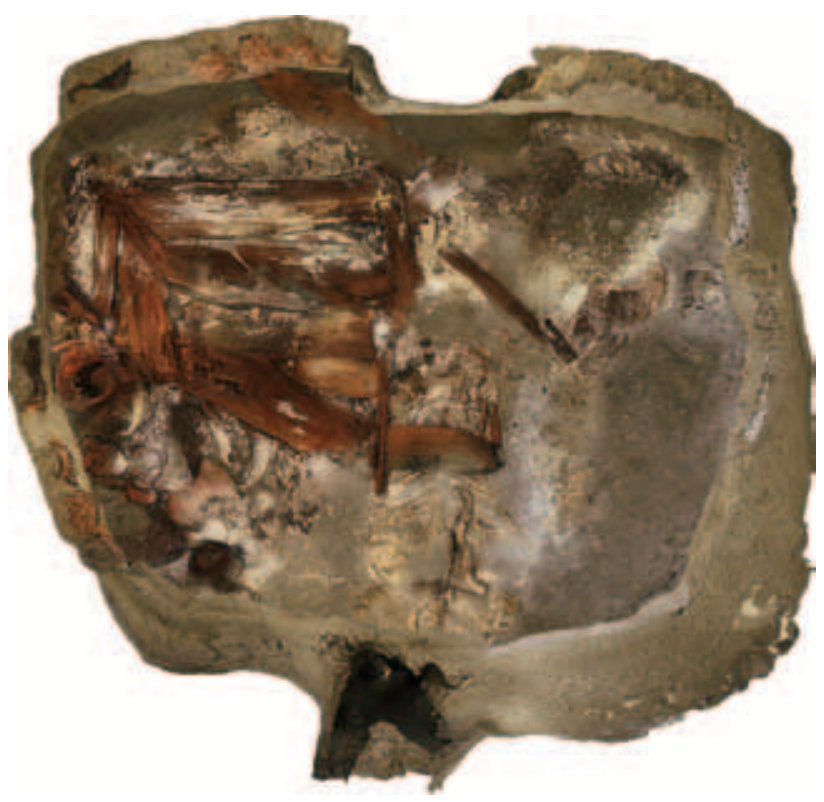

Figure 16 The chamber of Chuvthag 2014M2 (top is northeast).

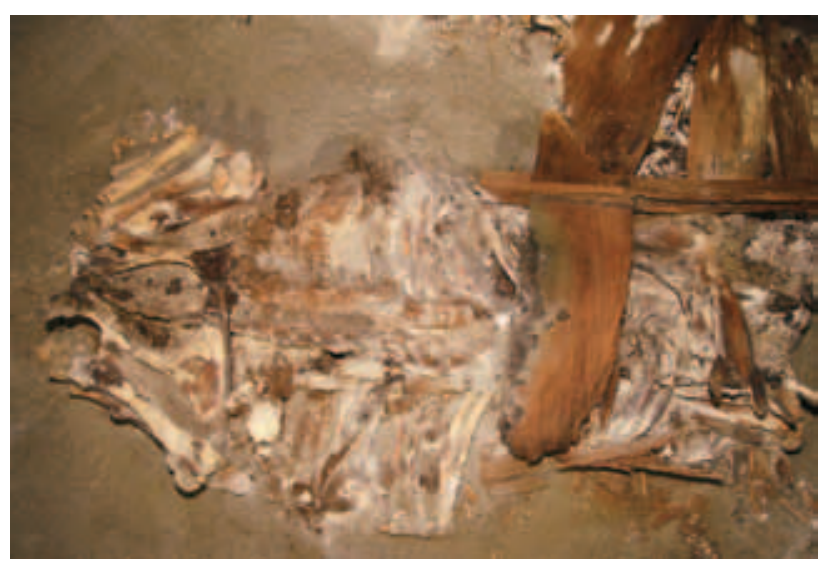

Figure 17 Horse burial in Chuvthag 2014M2 (SE-NW). low the wooden coffin there were stone boulders arranged to form a coffin bed that originally supported the wooden coffin together with a number of animal sacrifices. Because the animal carcasses had decomposed, the wooden coffin was tilted and the human remains and grave goods inside had shifted. The burial occupant was placed on side and the face was covered with cloth. A wooden comb was placed on the chest and the body was covered in fabric but it was severely decayed and the original material and shape could not be identified. There was a small pottery jar on top of the head and there were a large number of small braids surrounding the head. At the feet, there were a large number of bronze ornaments, likely applications sewn onto the clothes. Underneath the body there was a chain of agate beads. Close to the left wall there were a wooden basin, basketry items, and a set of pottery vessels encompassing one large jar, four medium-sized jars, and one small jar. The opening of the large pottery jar was covered with a piece of cloth. A pottery jar that had been used for cooking had been places on a stove built of four stone boulders, and an iron rod was placed inside the jar. Below the wooden coffin and around it a large number of goat/sheep heads and the hide and fur were still preserved. Across from the opening of the burial chamber there was a complete horse with the horse head facing inward that had been buried under the sides of the collapsed coffin. In its mouth there was an iron horse bit and by the neck on the right side there was a wooden stick, potentially a horse whip (Figure 17). On the right side of the chamber similar rope-like objects and a small wooden barrel were found. As the burial chamber interior was humid, the objects and bones were all severely decomposed and covered with a thick layer of white substance.

$2014 \mathrm{M} 3$ was located $2.8 \mathrm{~m}$ south of $2014 \mathrm{M} 2$. The opening of the passage was flat and rectangular, oriented at $225^{\circ}$, and measured $2.3 \times 0.7 \times 4.7 \mathrm{~m}$. East of the opening of the passage on the soil surface a simple altarlike structure of piled-up stones was found on which a spouted pottery vessel with goat/sheep bones around it was found, likely the remains of sacrificial activities related to the burial (Figure 18). Near the opening of the passage the walls on both sides carried indentations left by ropes. The upper part of the passage was filled with pebbles and sandy soil that were rather loose. About $2.2 \mathrm{~m}$ below the opening there was a second-level ledge covered by stone slabs (Figure 19); below these slabs was the empty space without soil. Close to the western side there were covering stones placed in disarray that had likely been rearranged at a later time. On the bottom of the passage, two stone slabs were found that may have fallen down from the second-level ledge when the grave was reopened. The walls on both sides of the passages had two rows of 5-8 hollows each. On the eastern side of the passage the burial chamber was dug. The burial chamber entrance was slightly arched, $0.64 \mathrm{~m}$ wide and $1 \mathrm{~m}$ high. Between the left and the right side of the burial chamber there was a partitioning wall of $2.1 \mathrm{~m}$ width and $0.75 \mathrm{~m}$ thickness to separate the burial chamber into two abreast 
rooms (Figure 20). The left room was semicircular with a flat top and of $2.3 \mathrm{~m}$ width, $3.8 \mathrm{~m}$ depth, and $1.8 \mathrm{~m}$ height. Between the wall and roof there was a series of small recesses which contained basketry utensils or small pottery jars with food items. The right and left grave rooms were largely symmetrical, having a width of $2.1 \mathrm{~m}$, a depth of $3.6 \mathrm{~m}$, and a height of $1.7 \mathrm{~m}$. Inside the left room there was a wooden coffin (coffin no. 1). The coffin had collapsed and below the coffin there was a coffin bed made of stone boulders with animal bones around. Inside the coffin there was a painted wooden tray with a lid and a rectangular wooden plate. On the chest of the burial occupant there was a red agate bead and a large number of glass beads were arranged around the hips. The bones of the burial occupant were largely decomposed and incomplete; on the skull remains of a large number of thin braids could be identified, and the body likely had been placed in a flexed position heading west. Close to the left wall of the burial chamber there was a group of pottery jars comprising one large jar, five medium-sized jars and two small jars, most of them having been placed in a recess cut into the grave wall. Below the large jar there was a round hollow with an iron arrowhead inside. Outside the rectangular pit there were three stones forming a hearth on which a cooking pottery jar was placed. Below the wooden coffin and between the coffin and the wall of the burial chamber there were a large number of animal bones including cattle, horse, and sheep/ goat with wooden sticks, iron horse bits, thin iron rods, basketry items, and rectangular wooden trays (Figure $21)$. In the right chamber two wooden coffins were found that are addressed as coffins no. 2 and no. 3 (Figure 22). Coffin no. 2 already collapsed and the wooden boards were in disarray. Below the coffin there was a coffin bed built of stone boulders and a large number of animal bones. Inside the coffin, the occupant was placed in a flexed position heading north. Next to the body there were basketry items, pottery jars, rectangular wooden

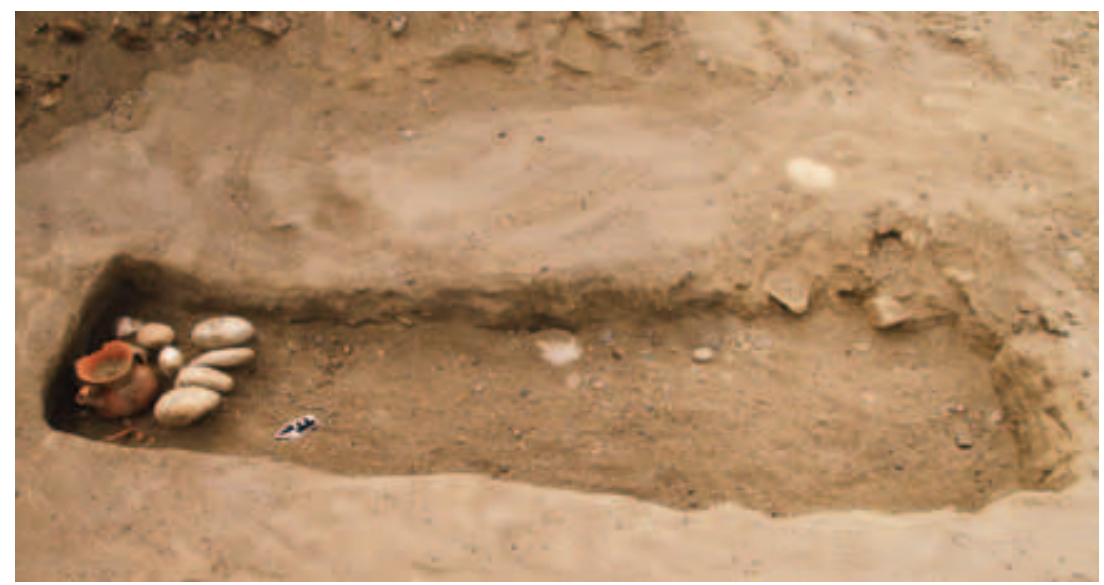

Figure 18 Sacrificial activity traces at opening of the passage of Chuvthag $2014 \mathrm{M} 3$ (NW-SE).

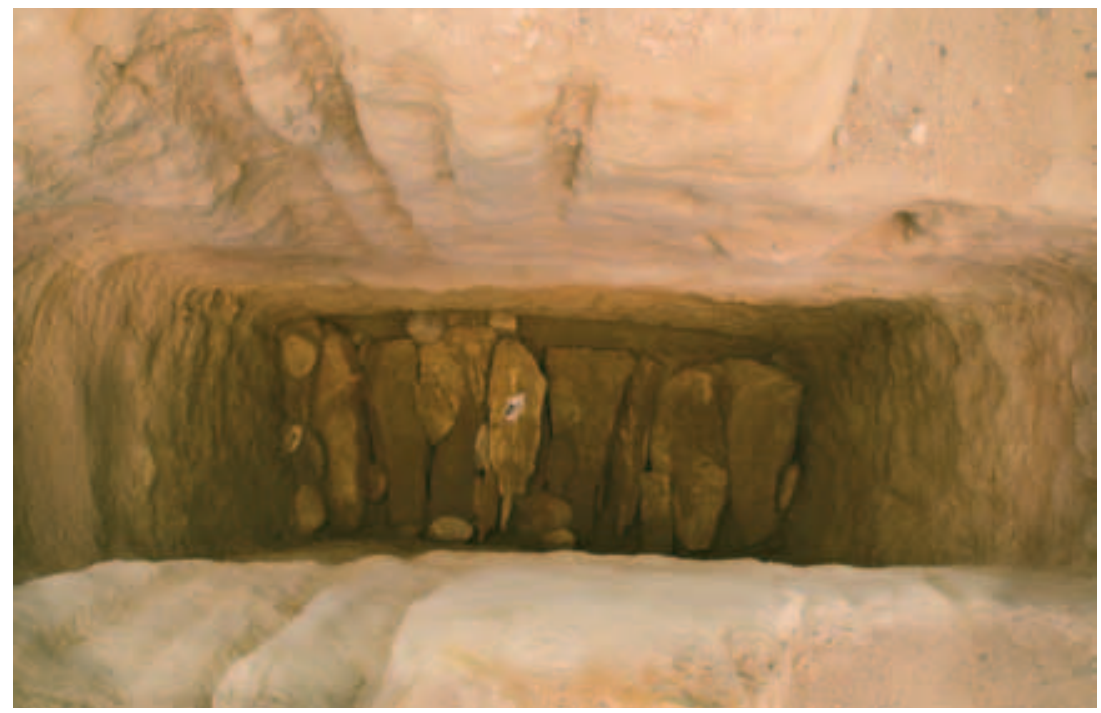

Figure 19 Stone cover slabs of second-level ledge of Chuvthag 2014M3 (SENW).

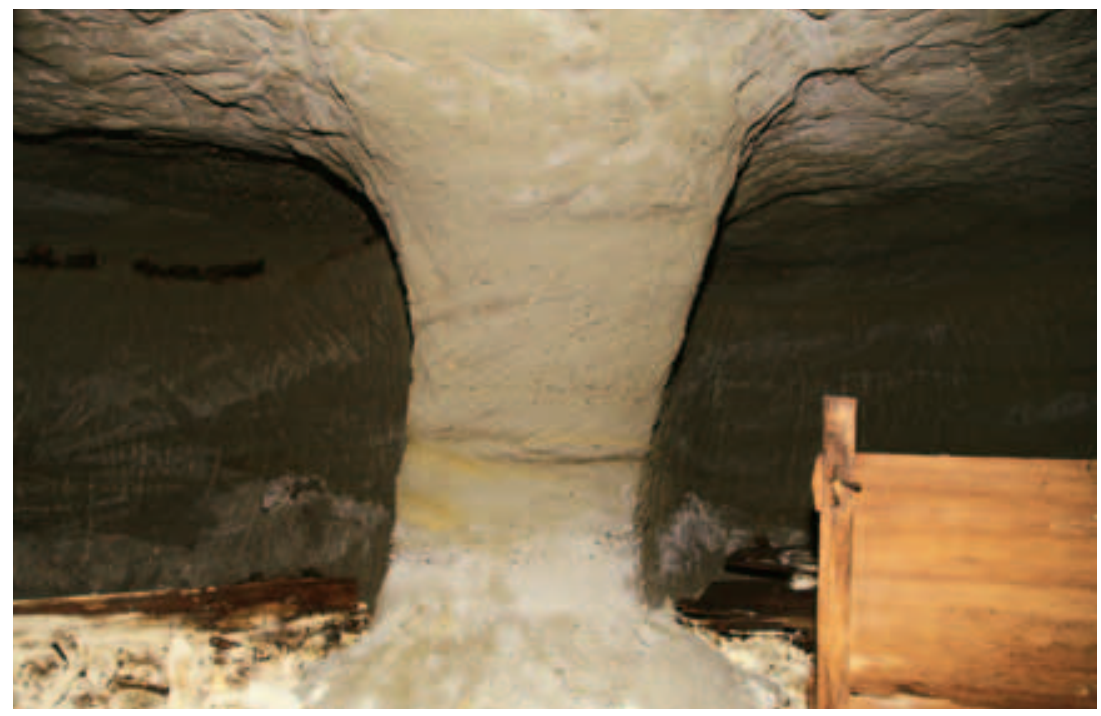

Figure 20 Left and right chambers of Chuvthag 2014M3 (SW-NE). 


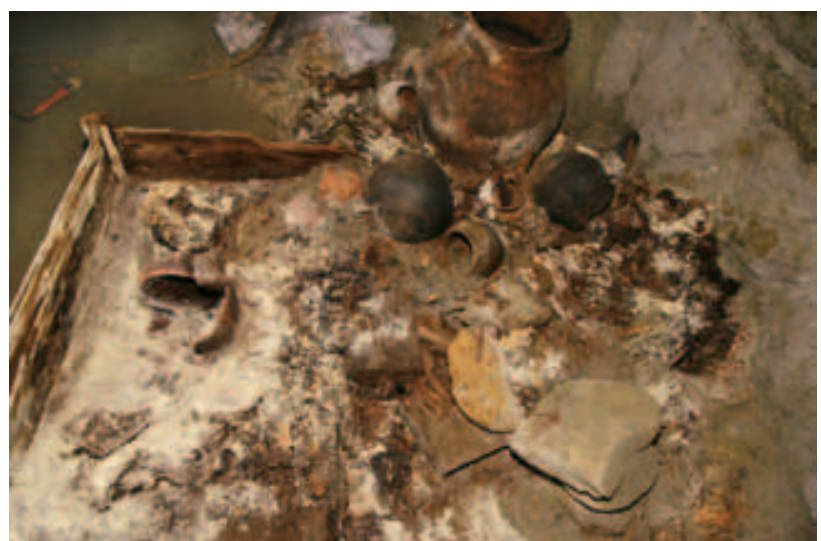

Figure 21 Grave goods in the left chamber of Chuvthag 2014M3 (NE-SW).

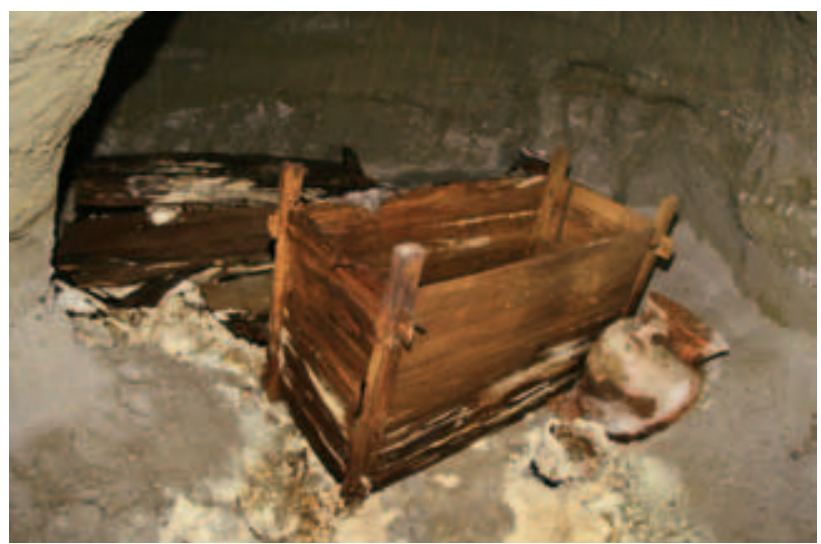

Figure 22 Wooden coffins in the right chamber of Chuvthag 2014M3 (NW-SE).

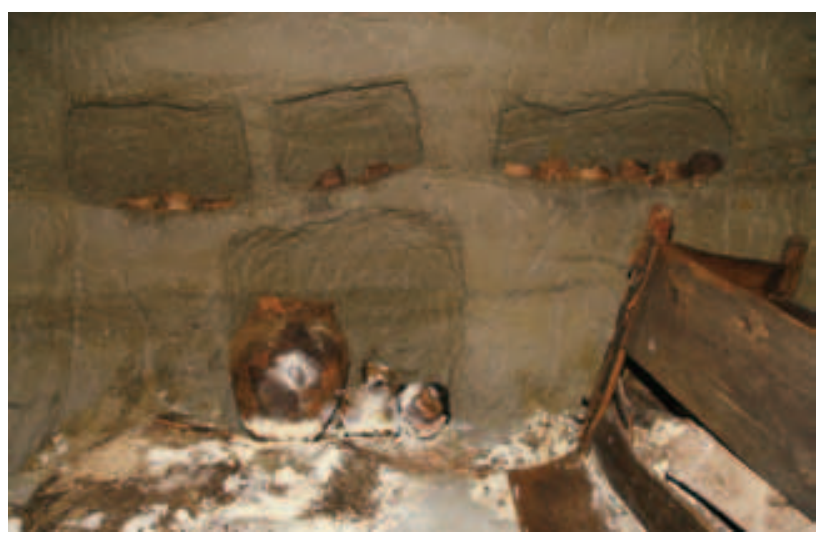

Figure 23 Rear wall of Chuvthag 2014M4 (SW-NE).

trays, engraved wooden plaques, round shell ornaments, and a bronze bracelet worn around the wrist. Next to the right side of the chamber there was a set of pottery jars placed into a rectangular hollow. The set comprised one large jar and three medium-sized jar. Inside the chamber on the bottom there were a large number of animal bones, including horse, sheep/goat, and cattle bones. Between the animal bones there was a wooden-handled iron dagger, an iron belt buckle, basketry items, and iron arrowheads. At the grave entrance there was a cooking pot with stones supporting it as well as a bamboo bow, iron arrowheads, basketry items, a small wooden bucket, and a complete sheep/goat skeleton. Coffin no. 3 was positioned in between coffin no. 2 and the burial chamber entrance, overlaying the animal offerings and grave goods of coffin no. 2 . The wooden coffin was rather well preserved, had no lid, but was covered with cloth of which a small number of fragments remain as well as traces on the top of the coffin. The human bones inside the coffin are rather well preserved and the head was placed in the north with traces of a large number of small braids on it. The clothes were largely decomposed. On the body there were fragments of a wooden comb, pottery jars, and a rectangular wooden tray. The other grave goods had been placed between the wooden coffin and the wall of the chamber, including a large pottery jar and a pottery cooking pot with clear traces of soot on it and a wooden stick inside. Based on the preservation conditions, the stone covering slabs placed on the second-level ledge, and the stone slabs fallen onto the bottom of the passage, coffin no. 3 was likely placed into the grave as a secondary burial after the grave had been reopened.

$2014 \mathrm{M} 4$ was located $3.6 \mathrm{~m}$ south of $2014 \mathrm{M} 3$. The opening of the passage was rectangular in form and disturbed by a ditch in the western corner. It was oriented at $211^{\circ}, 1.8 \mathrm{~m}$ long, $0.6 \mathrm{~m}$ wide and $4.1 \mathrm{~m}$ deep. Close to the entrance there were deep indentations made by ropes on both walls. The upper part of the passage was filled with pebbles and loose sandy soil. At a depth of $2.3 \mathrm{~m}$ on the left and right sides there was a second-level ledge covered with stone slabs. Underneath the stone slabs was hollow space without any soil. On the walls of the passage there are deep hollows for climbing. On the bottom of the passage the burial chamber was opened toward the east. The entrance of the burial chamber was arched, $0.6 \mathrm{~m}$ wide and $0.9 \mathrm{~m}$ high. The chamber was rectangular in shape, $2.65 \mathrm{~m}$ wide, 2.55 deep, and $1.65 \mathrm{~m}$ high. On all four walls of the burial chamber there were several small recesses; on the rear wall there were three rectangular recesses that contained basketry items. In the lower part of the rear wall there was a large square recess; on the floor, clay bars were used to build a frame of about the same size as the recess; inside that frame, a group of pottery wares were placed including one large jar and four medium-sized jars (Figure 23). On the upper portion of the wall on the left side of the burial chamber there was one rectangular recess and on the upper part of the left side there were two rectangular recesses, all of them containing basketry items. On the left side of the burial chamber there was a horse heading inward; between the horse head and the rear wall, a number of items had been placed including ropes, twigs, sheep/goat bones, a large number of barley seeds, and on the pile of barley seeds the traces of woven organic material, likely the remains of a bag that contained the grains. At the rear wall in front of the recess there was a bundle of branches that may have been meant 
to serve as fuel. On the right side of the burial chamber a rectangular box-like wooden coffin has been found, one of its sides being fragmented but not completely disintegrated, however, the lid had already collapsed into the coffin. Based on the restoration, the wooden coffin measured $1.2 \mathrm{~m}$ in length, $0.58 \mathrm{~m}$ in width, and $0.7 \mathrm{~m}$ in height, and the wooden coffin boards were $1.2 \mathrm{~cm}$ thick. On the lid, pottery jars, a rectangular wooden tray, and many basketry items had been placed; between the wooden coffin and the right wall of the chamber there was one small pottery jar that had probably fallen down from the coffin lid. A large number of objects were piled up inside the coffin including four wooden weaving tools, two color-painted wooden trays, bundles of twigs, iron bracelets, etched agate beads, red agate beads, fragments of wooden combs, handled bronze mirrors, engraved wooden plaques, small pottery jars, rectangular wooden trays, basketry items, and a sheep's head (Figure 24). The burial occupant was placed in a flexed position heading east and fragments of many small braids remaining on the skull; there were remains of clothing on the body but they were largely decomposed. On the body there were bronze beads, bronze bells, and on the arms and hips there were a large number of bronze beads and bronze fragments that were probably part of the clothing ornaments. On the bottom of the coffin there was a layer of felt-like bedding and between them and the bottom boards of the coffin there was a layer of cereal seeds. Beneath the coffin, stones had been piled up to form a coffin bed and there were a large number of animal bones as well (Figure 25). Between the wooden coffin and the entrance of the burial chamber there were a large number of sheep/goat bones. Near the right wall of the chamber there was one large white stone slab and a number of small stones that were likely connected to some ritual customs related to stone-erecting. On the rear wall above the small recess there were simple engraved depictions of animals, probably two horses with their head pointing to the right and on their right an engraved folding ruler-like image.

2. Unearthed artifacts

The six burials excavated at Chuvthag Zone II had not been robbed and were rather well preserved. A large number of grave goods have been unearthed that fall into the categories of pottery wares, wood and bamboo objects, gold, bronze, iron, basketry items, bone objects and textiles.

(1) Pottery wares: 79 items, all in the form of jars. According to shape and function, we can classify them into six types.

Type A: nine items. 2014M5 (single interment) held two jars of Type A; in all other interments each burial occupant had one. All of them are made of red coarse sandy ware. They are rather large with a wide opening, high neck, sloping shoulder, an oblique body, and a round bottom. On the shoulder there are two handles, some of them with small knobs on them and with incised decors on the back. Below the shoulder, the vessels are decorated with coarse cord mark pattern, and some have vertical wave

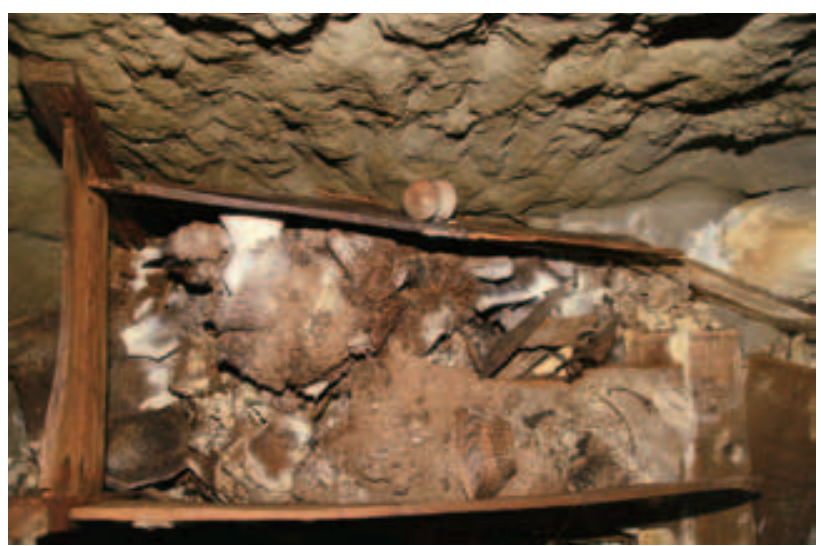

Figure 24 Wooden coffin of Chuvthag 2014M4 (top is southeast).

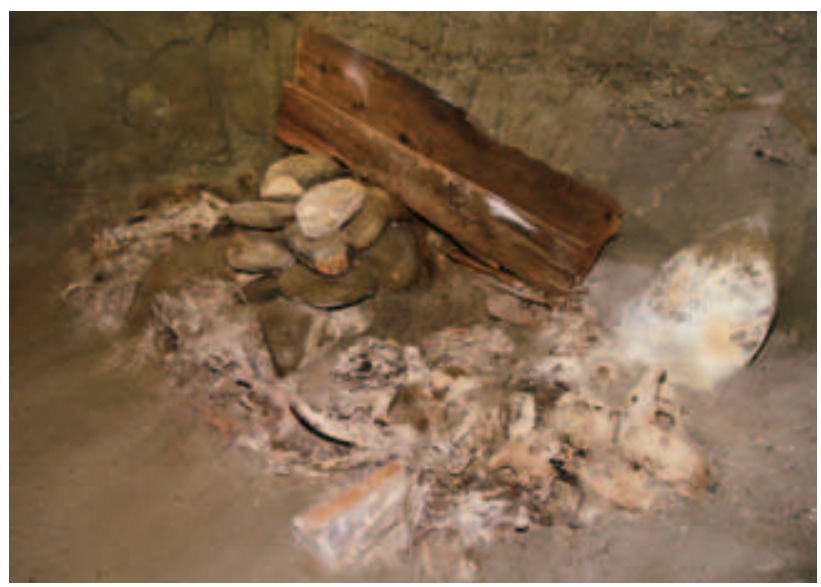

Figure 25 Accumulations underneath the wooden coffin of Chuvthag 2014M4 (W-E).

pattern on the neck. Most of them had been covered with a piece of cloth at the time of unearthing and there were wooden sticks inside them, suggesting that they may have been used to store solid food. The jar 2014M4:2 had a rim diameter of $30.4 \mathrm{~cm}$ and was $64.3 \mathrm{~cm}$ high (Figure 26).

Type B: nine vessels, red coarse sandy ware. They have a wide outward-flaring opening, long neck, and round bottom. There are two handles extending from neck to shoulder, some of them having a horn-shaped application, and the handles carry incised decors on their backs. On the necks there are reddish-brown painted patterns, most of them vertical waves, and the bodies are decorated with cord mark pattern; some vessels carry painted spiral pattern and appliqué bands around the neck. Based on overall shape, we can distinguish two subtypes.

Subtype BI: six items. These vessels have a longer neck and a round belly. 2014M5:11 has a rim diameter of $17.8 \mathrm{~cm}$, a bottom diameter of $3.6 \mathrm{~cm}$, and a height of $22.7 \mathrm{~cm}$ (Figure 27).

Subtype BII: three vessels. Their bellies are wider. $2014 \mathrm{M} 2: 2$ has a rim diameter of $14 \mathrm{~cm}$ and a height of $24 \mathrm{~cm}$ (Figure 28). 


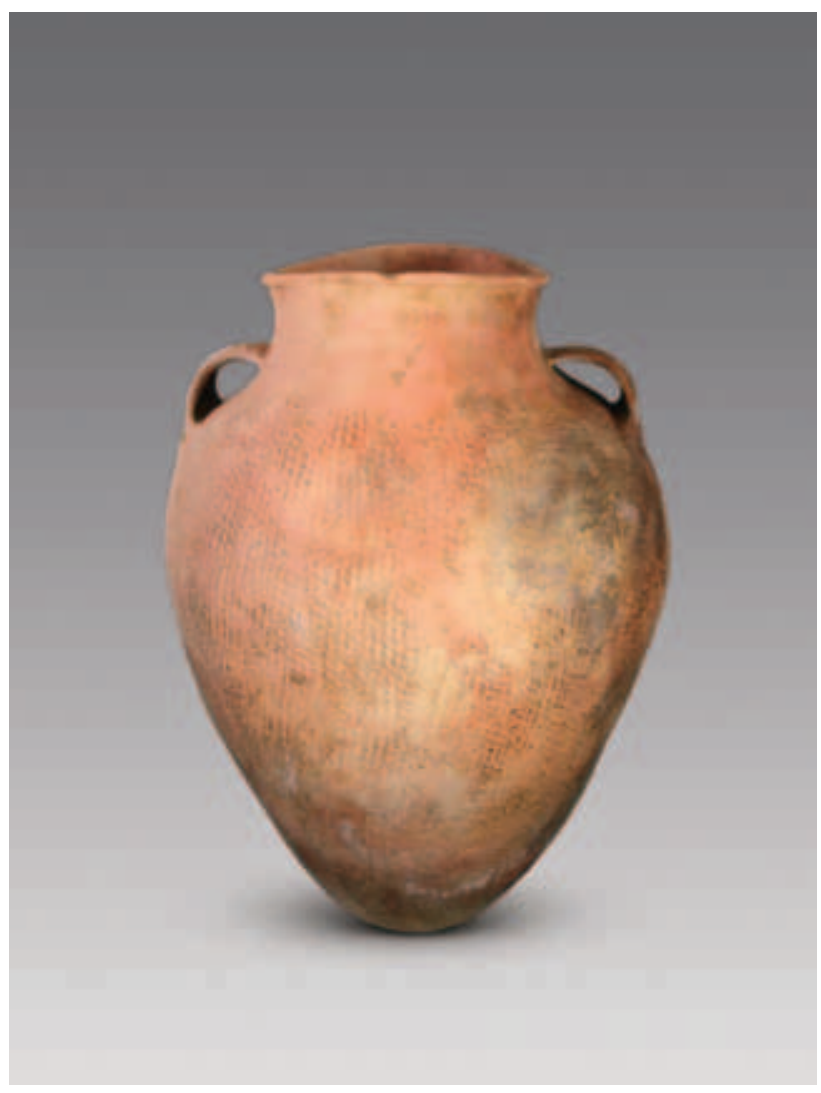

Figure 26 Pottery jar, Type A (Chuvthag 2014M4:2).

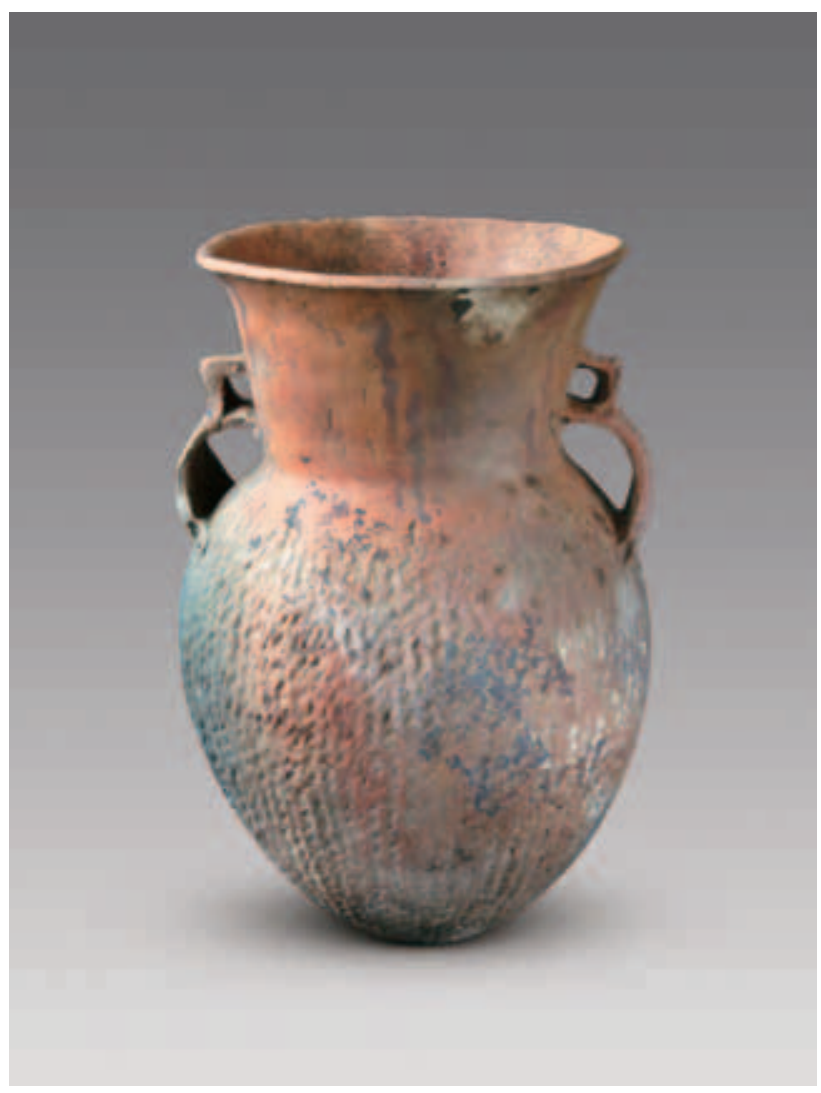

Figure 28 Pottery jar, Type BII (Chuvthag 2014M2:2).

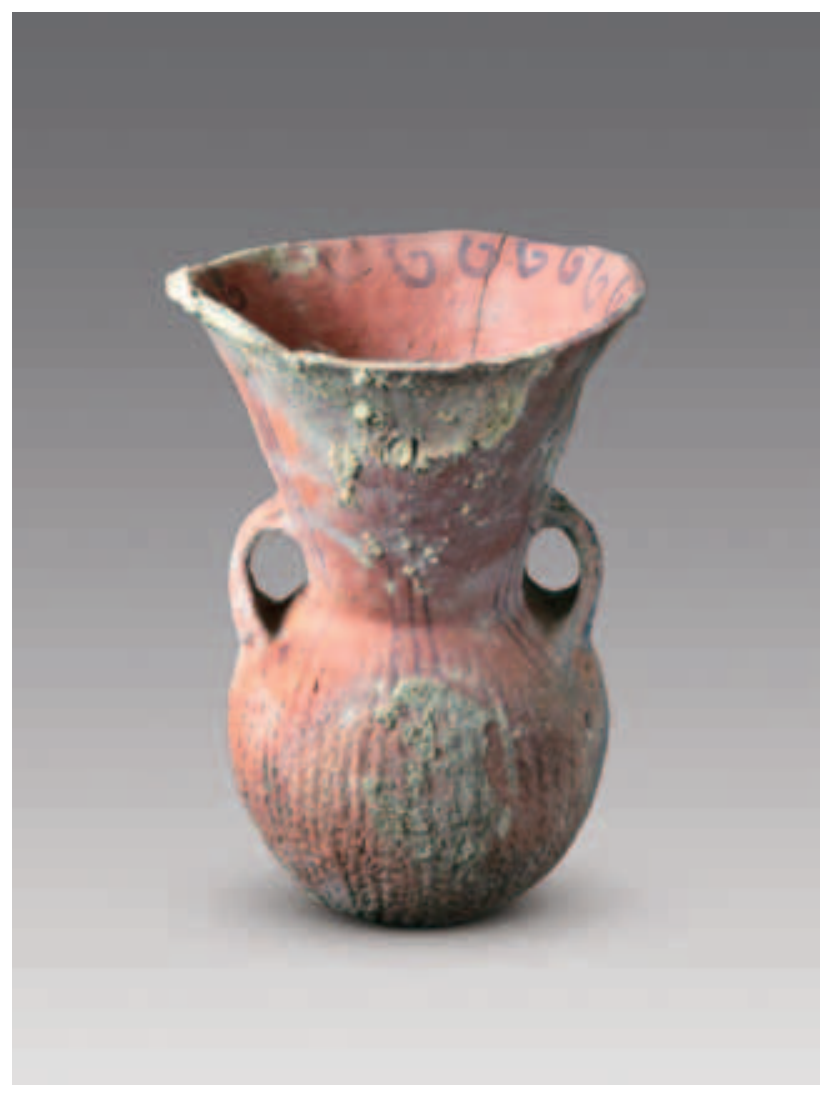

Figure 27 Pottery jar, Type BI (Chuvthag 2014M5:11).

Type C: five items. These vessels have a spout and are made of red coarse ware. They have a wide opening, high neck, globular belly, and round bottom. The spouts are long and tubular with a variety of different cross-section forms. On the other side of the spout, they have a single handle that carries a small horn-shaped application on top. Below the shoulder there are circular or triangular incised patterns. On neck, body, and spout there is painted decoration, mostly in the form of wave pattern, or incised patterns along the rim. 2014M3 left:9 has a rim diameter of $13.2 \mathrm{~cm}$, a bottom diameter of $4.6 \mathrm{~cm}$, and a height of $16 \mathrm{~cm}$ (Figure 29).

Type D: six items, spouted jars made of red coarse sandy pottery. They have wide outward-flaring openings and the rim on one side is pinched into an inclined chutelike spout; they have a globular body and round bottom. The body is decorated with cord mark pattern. Based on the overall form, we can distinguish two subtypes.

Subtype DI: three items that have a long neck and spout. Below the spout between neck and shoulder there is a bridge-shaped knob and on the opposite side of the spout there is a handle with a horn-shaped application on its upper part. 2014M3 right:2 has a rim diameter of $13.2 \mathrm{~cm}$, a bottom diameter of $4 \mathrm{~cm}$, and a height of $19.2 \mathrm{~cm}$ (Figure 30).

Subtype DII: three items with a short neck. On the opposite side of the spout there is a single vertical handle with incised pattern on it. Some of the samples have soot 
traces on them. 2014M3 right:7 has a rim diameter of $12.2 \mathrm{~cm}$ and a height of $15.1 \mathrm{~cm}$ (Figure 31 ).

Type E: 18 double-handled jars made of red coarse wear. They can be classified into two subtypes.

Subtype EI: 16 items of coarse quality with cord mark pattern below the shoulders. On the surface there are thick layers of soot, and most items of this subtype had an iron or wooden stick inside them. Below the jars or close to them three or four stones had been placed, indicating that they were meant for cooking. Sample 2014M2:1 has a rim diameter of $16 \mathrm{~cm}$ and a height of $18.3 \mathrm{~cm}$ (Figure 32 ).

Subtype EII: two items of higher quality with knobs on the two handles and decoration on neck and shoulders. 2014M3 right: 1 has rope-shaped appliqué bands on opening and neck and fret pattern on the body. The rim measures $10.7 \mathrm{~cm}$ in diameter and the jar is $13.5 \mathrm{~cm}$ high (Figure 33).

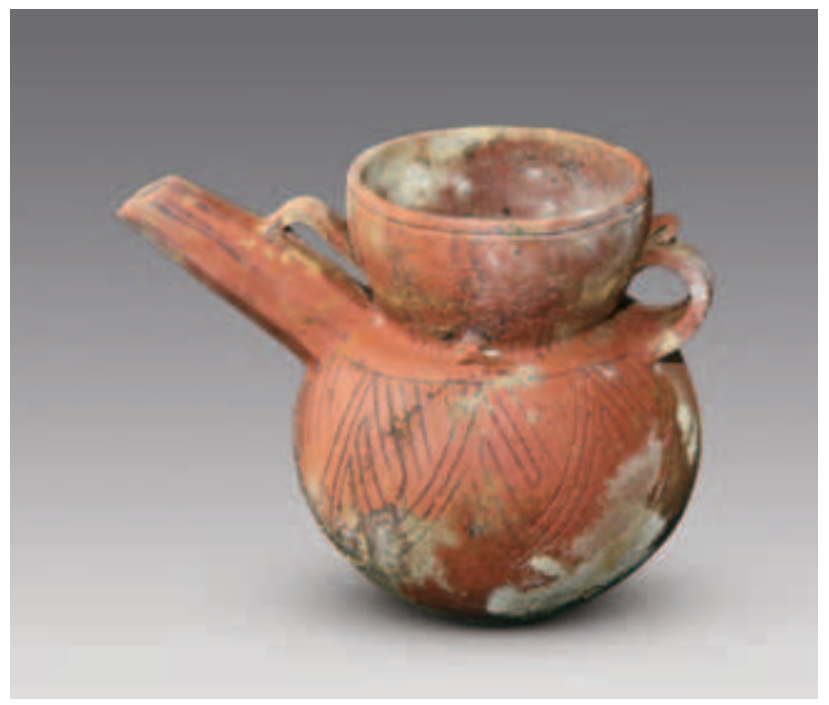

Figure 29 Pottery jar, Type C (Chuvthag 2014M3 left:9).

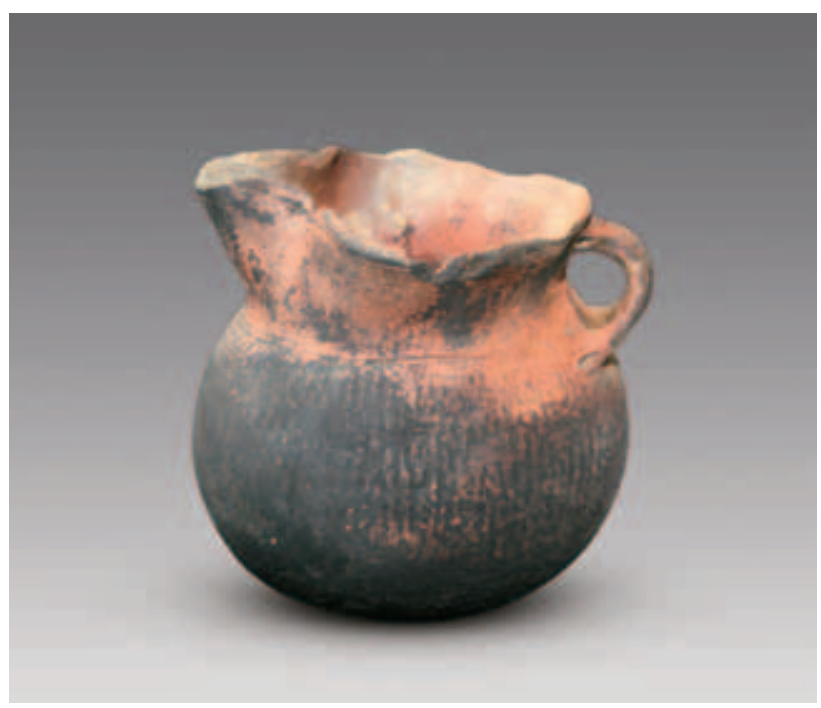

Figure 31 Pottery jar, Type DII (Chuvthag 2014M3 right:7).
Type F: 32 single-handled jars. They are rather small and elaborately made. They can be classified into four subtypes.

Subtype FI: eight items with straight opening, high neck, single handle, sloping shoulder, and round bottom. The necks are decorated with painted wave pattern, the shoulders carry line pattern, and below the shoulders there is cord mark pattern. 2014M2:3 is a red fine clay ware. Its rim diameter is $8 \mathrm{~cm}$, the bottom diameter is $3.8 \mathrm{~cm}$, and the jar is $7.4 \mathrm{~cm}$ high (Figure 34 ).

Subtype FII: seven items. They have a wide trumpetshaped mouth, a handle on one side, a globular body, and a round bottom. The bellies are decorated with incised fret pattern, string pattern, or not decorated at all. 2014M3 right:5 is a red coarse sandy ware. The rim diameter measures $8 \mathrm{~cm}$ and the vessel is $8.9 \mathrm{~cm}$ high (Figure 35 ).

Subtype FIII: five items with a single handle, no neck,

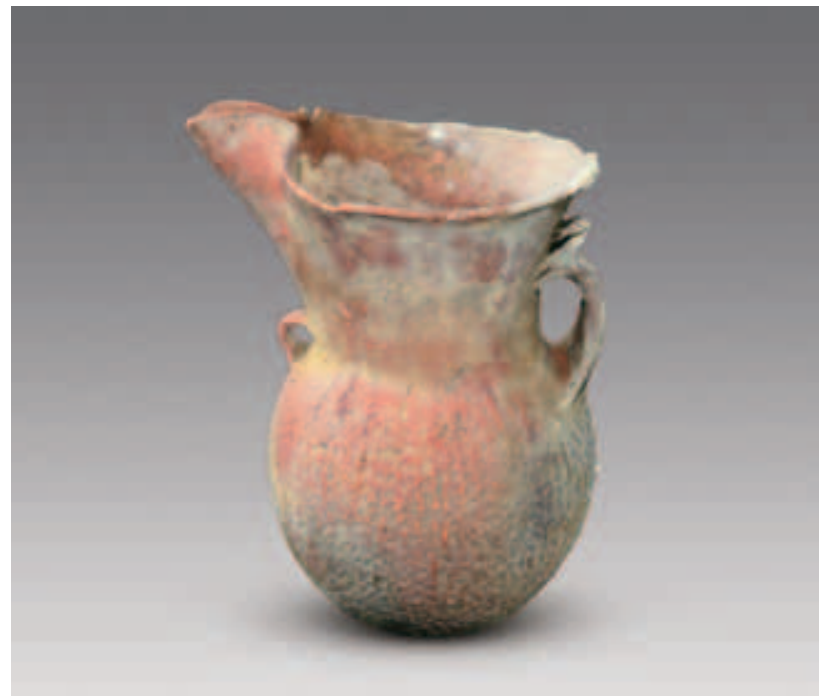

Figure 30 Pottery jar, Type DI (Chuvthag 2014M3 right:2).

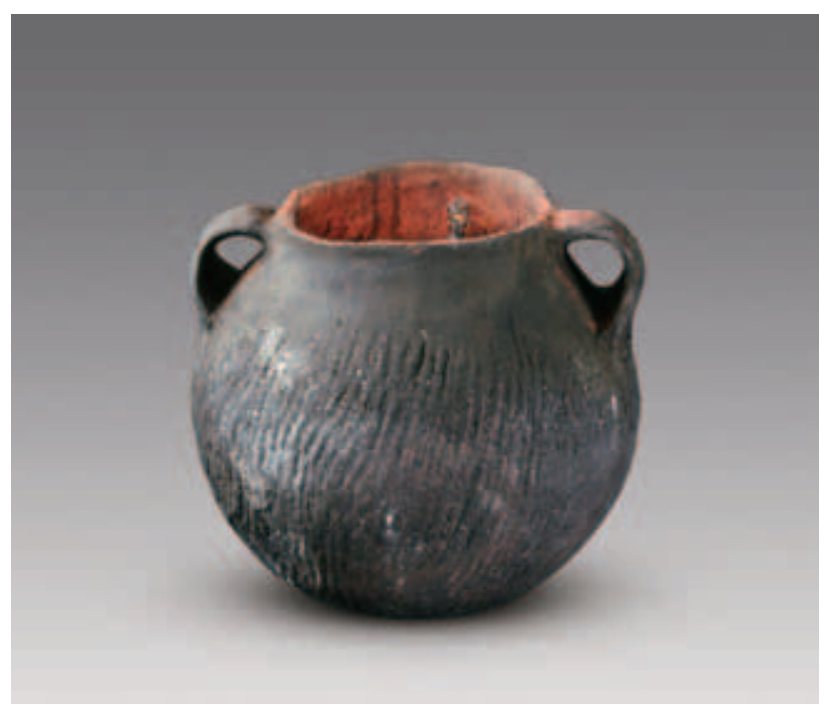

Figure 32 Pottery jar, Type EI (Chuvthag 2014M2:1). 


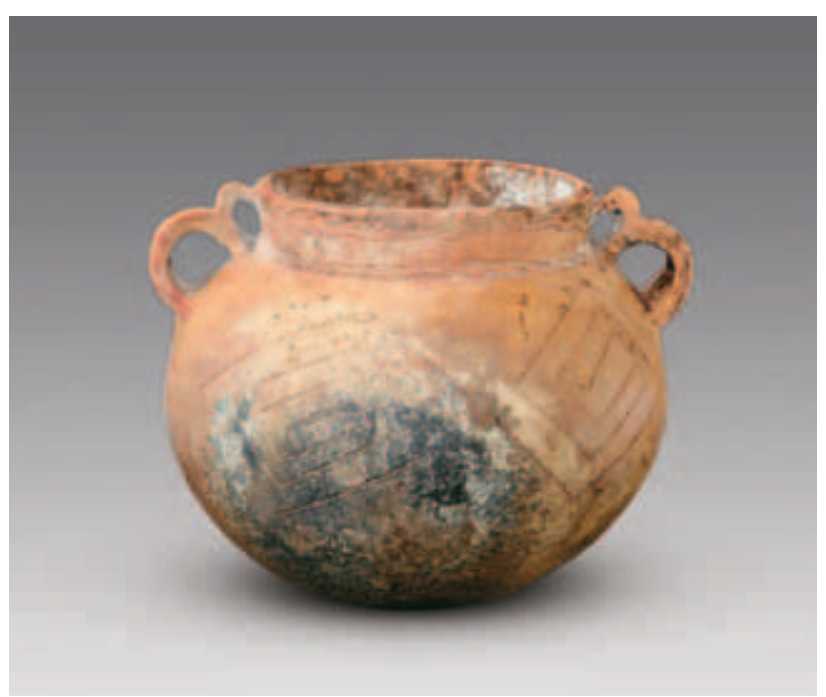

Figure 33 Pottery jar, Type EII (Chuvthag 2014M3 right:1).

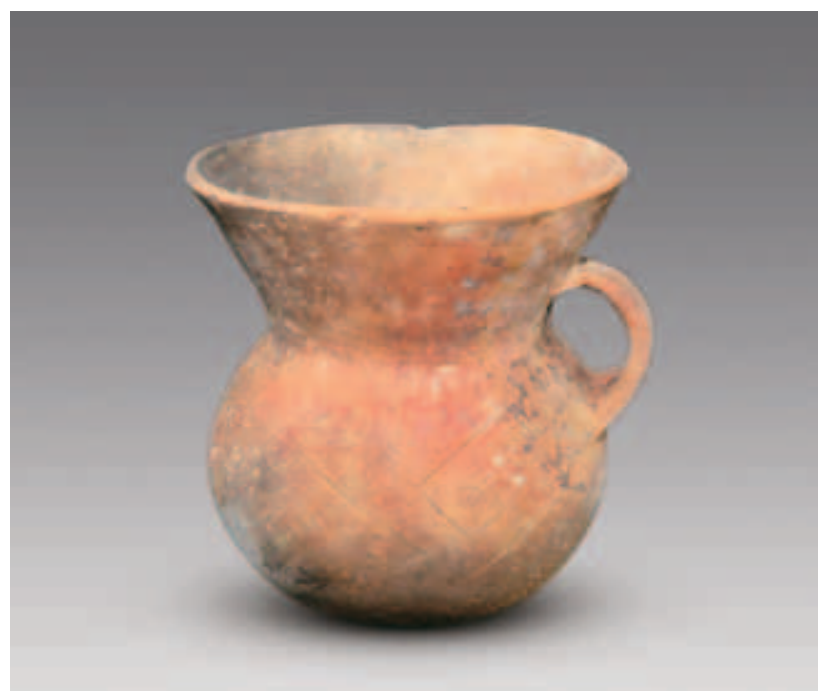

Figure 35 Pottery jar, Type FII (Chuvthag 2014M3 right:5).

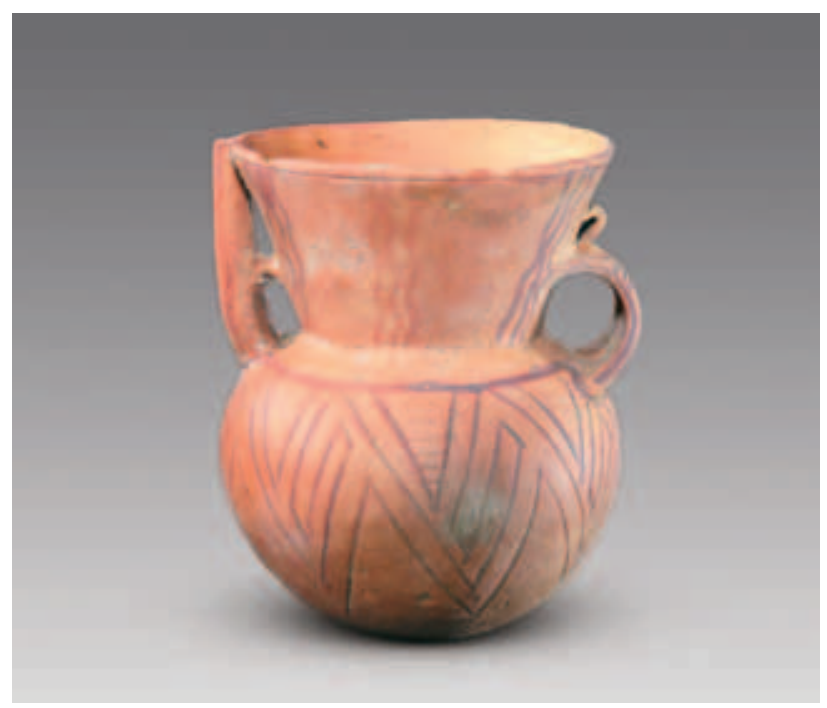

Figure 37 Pottery jar, Type FIV (Chuvthag 2014M3 left:3).

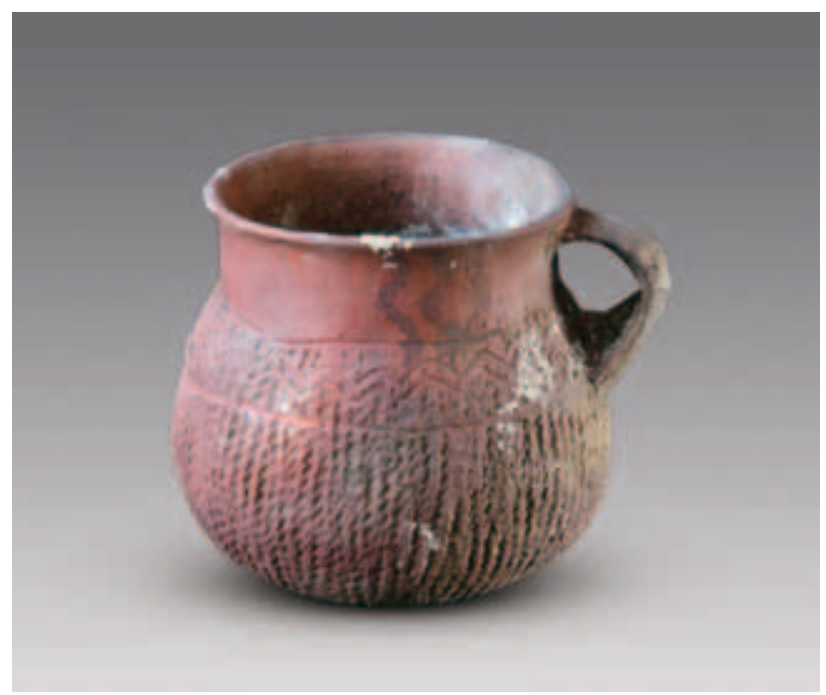

Figure 34 Pottery jar, Type FI (Chuvthag 2014M2:3).

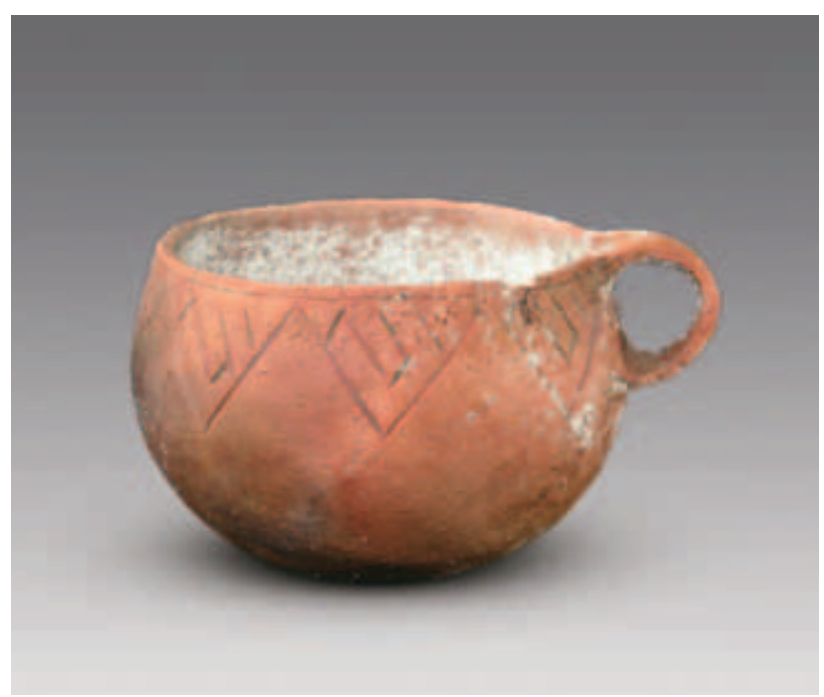

Figure 36 Pottery jar, Type FIII (Chuvthag 2014M2:4).

and a round bottom. Below the rim the bodies are decorated with incised triangle pattern, wave pattern or string pattern. 2014M2:4 is a red fine clay ware. It has a rim diameter of $9.2 \mathrm{~cm}$, a bottom diameter of $3 \mathrm{~cm}$, and a height of $6.4 \mathrm{~cm}$ (Figure 36 ).

Subtype FIV: 12 vessels made of red fine ware. They have a trumpet-shaped mouth, high neck, single handle, a knob on the base of the handle, a straight handle on the opposite side at the neck, and a globular body. On the body there are incised triangle and fret decors painted with reddish-brown color; the neck carries red-brown painted vertical wave pattern. 2014M3 left:3 has a rim diameter of $8.1 \mathrm{~cm}$, a bottom diameter of $2.3 \mathrm{~cm}$, and a height of $10.4 \mathrm{~cm}$ (Figure 37 ).

(2) Bamboo or wooden objects: 44 items including trays, basins, textile-weaving tools, engraved wooden plaques, combs, bows, etc.

Four-legged wooden trays: four items. 2014M4:9 has 
a four-legged cauldron shape. The tray surface is of a flat round shape and filled with tea-like food remains. It has two upright handles, the four legs point outward and they are reinforced by cross-shaped ties. The outside of the tray is coated with reddish-brown color and painted with zigzag pattern. On the roots of the four legs there are painted patterns: one is painted with gold ground, on which the right side carries opposite $\mathrm{S}$-shaped patterns drawn in ink lines with a yak head and an ox head with a flying bird in front of it painted in black ink. The ornaments below the bird are unclear. One foot carries a pair of blue sheep outlined in gold color; another foot carries birds back to back outlined in gold powder, and below the birds there is an unclear gold shape. The fourth foot has been severely damaged and the design on it is unidentifiable. The feet of this tray were already broken at the time of interring and bore small holes carrying thread remains, likely the traces of an earlier repair. The upper diameter is $17.2 \mathrm{~cm}$, the feet are $14.6 \mathrm{~cm}$ high, and the overall height is $22.9 \mathrm{~cm}$ (Figure 38). 2013M1:20 was carved out of one piece of wood; the tray is rectangular and concaved at the bottom. On one side there is a triangular protrusion that can be used to lift the tray; the one on the other side is broken. The four feet are thick and point outward on both sides. Both inside and outside there are traces of red and black lacquer paint. The extant length of the table is $22.5 \mathrm{~cm}$, the width is $16.7 \mathrm{~cm}$, and the full height is $19.5 \mathrm{~cm} .2014 \mathrm{M} 2: 12$ has a lid and the tray surface is square in form with straight handles on both sides that are decorated with incised fret pattern and red paint; the sides are decorated with wave pattern. The rim is decorated with a row of red line pattern as well as sets of vertical wave and lozenge pattern. The legs are square in cross section, thick on top and thin on the bottom. One leg is broken, one foot had been broken and was repaired as shown by holes and traces of thread. On the table there are blackish food remains. The side length measures $13.7 \mathrm{~cm}$, the legs are $10.6 \mathrm{~cm}$ high, and the full height is $19.6 \mathrm{~cm}$. The lid has two handles with four stick feet and slanted sides. The outside of the rim carries a read painted line, there is red line decoration on the handles, and on the outer walls on both sides there is further rod decoration. The lid is $8.8 \mathrm{~cm}$ long, $6.3 \mathrm{~cm}$ wide, and $11 \mathrm{~cm}$ high.

One square-footed wooden tray (2014M4:8): the tray surface is rectangular in the shape of a shallow plate. It has two arc-shaped handles, a round bottom, and below a square foot. On all four sides the foot is painted and the front carries a layer of gold color with two confronting peacocks on clouds painted in black ink lines; the other sides are painted with zigzag pattern, spiral pattern and other geometric patterns in black or gold. The side length at rim is $19.6 \mathrm{~cm}$, that at foot is $10.3 \mathrm{~cm}$, and the tray is $15 \mathrm{~cm}$ high (Figure 39 ).

20 wooden basins: they are all cut out of whole wood block, mostly birch. They are rectangular with handles protruding on both sides, and a slightly arched bottom. Most of the basins contained food remains. 2014M3 right: 15 is $30.8 \mathrm{~cm}$ long, $18.4 \mathrm{~cm}$ wide and $3.8 \mathrm{~cm}$ high

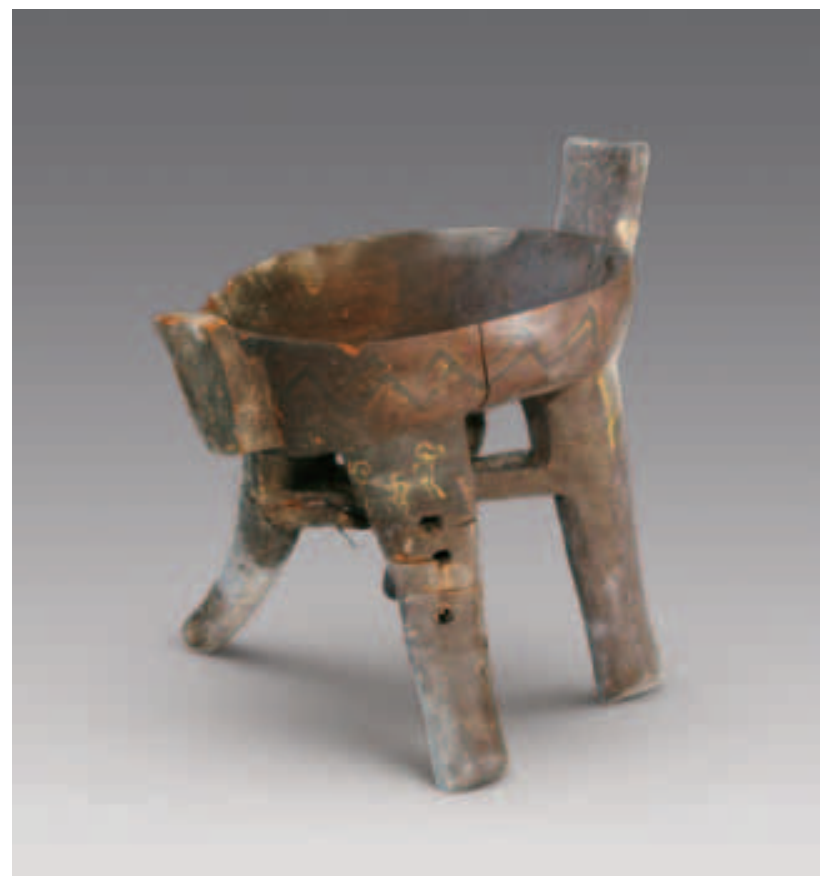

Figure 38 Four-legged wooden tray (Chuvthag 2014M4:9).

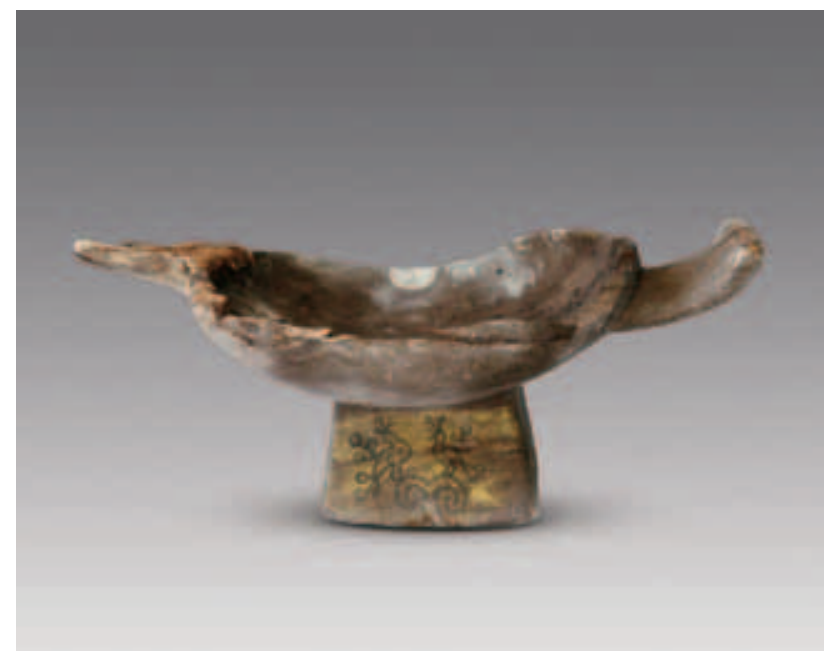

Figure 39 Square-footed wooden tray (Chuvthag 2014M4:8).

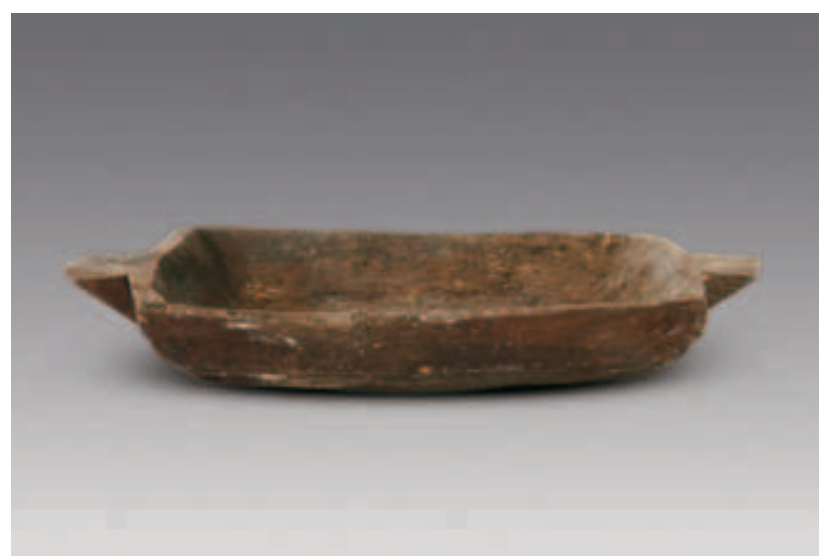

Figure 40 Wooden basin (Chuvthag 2014M3 right:15). 


\section{(Figure 40).}

Three wooden combs: they are rectangular in shape with narrow teeth incised on one side. 2014M3 right:16 is $14 \mathrm{~cm}$ long, $8 \mathrm{~cm}$ wide, 1.4 thick, and the teeth are $5.2 \mathrm{~cm}$ long (Figure 41).

Two engraved wooden plaques: 2014M3 right:10 is rectangular with a spherical knob on one end with a hole that has the remainders of a piece of rope inside. The object is engraved on both sides, one side carrying two full S-shapes and one incomplete one; the other side is decorated with a pair of stylized S-shapes connected at a point in between. The plaque is $4.5 \mathrm{~cm}$ long, $2.4 \mathrm{~cm}$ wide, $0.7 \mathrm{~cm}$ thick, and the hole diameter measures $0.2 \mathrm{~cm}$ (Figure 42). 2014M4:21 is rectangular with two holes in the middle that contains remainders of rope. The plaque is engraved on both sides, one carrying a checkered pattern and a stylized double S-shape; the other side is engraved with fret pattern on one side and unclear pattern on the other. The plaque is $6.1 \mathrm{~cm}$ long, $1.2 \mathrm{~cm}$ wide, and $0.5 \mathrm{~cm}$ thick;

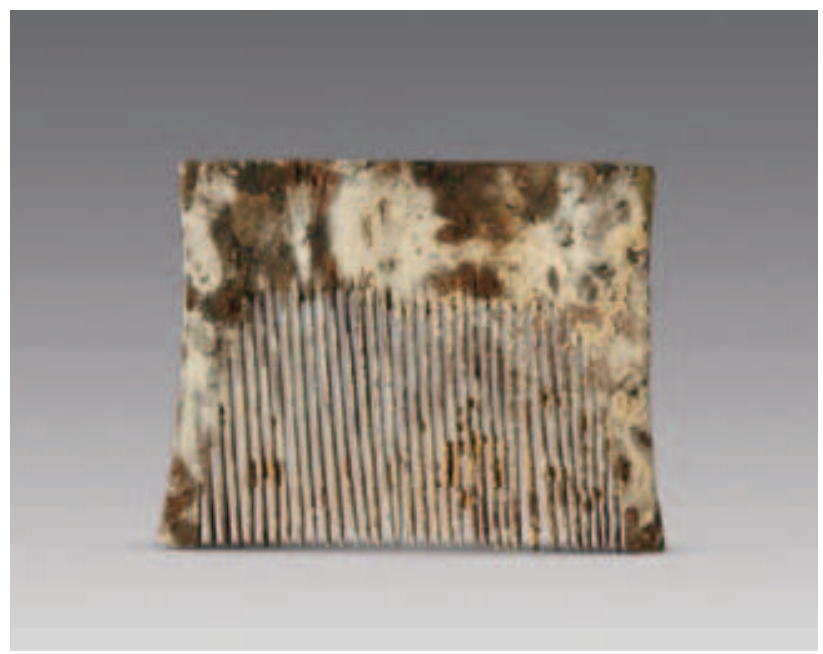

Figure 41 Wooden comb (Chuvthag 2014M3 right:16).

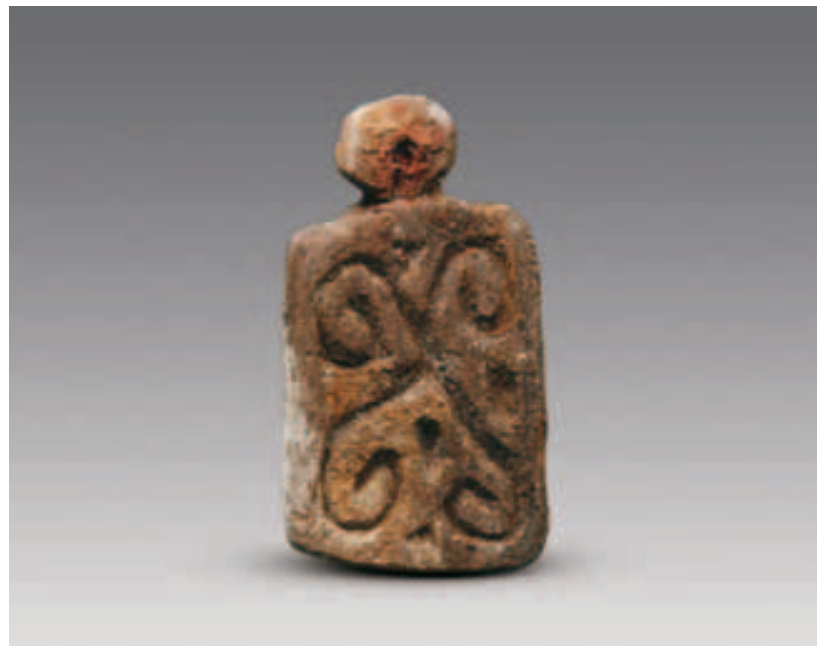

Figure 42 Engraved wooden plaque (Chuvthag 2014M3 right:10). the holes have a diameter of $0.2 \mathrm{~cm}$ (Figure 43 ).

Four wooden textile-weaving tools: all of them were found in burial 2014M4. 2014M4:16 is of a flat slat shape incised on both sides with narrow grooves that hold remains of silk yarns. The object is $34.7 \mathrm{~cm}$ long, $4.2 \mathrm{~cm}$ wide and $1.5 \mathrm{~cm}$ thick (Figure 44). 2014M4:17 has a semicircular cross-section with one handle on each end bent toward the same side with remains of silk yarns on them. The object is $32.6 \mathrm{~cm}$ long, $3.5 \mathrm{~cm}$ wide and $1.6 \mathrm{~cm}$ thick.

One barrel-shaped wooden object (2013M1:24): the object has a round lid with a mushroom-shaped grip in the top center; the vessel body is round barrel shaped with a flat bottom. The rim diameter measures $2.8 \mathrm{~cm}$, the bottom diameter measures $3.3 \mathrm{~cm}$, and the vessel is $9.7 \mathrm{~cm}$ high.

One wooden rake-shaped object (2013M1:25): this object consists of a thin wooden stick with a three-prong rake inserted into it and fastened with string. The object as a whole is $13 \mathrm{~cm}$ long and the prongs are $4.5 \mathrm{~cm}$ long.

One wooden stirrer (2013M1:26): the object was dis-

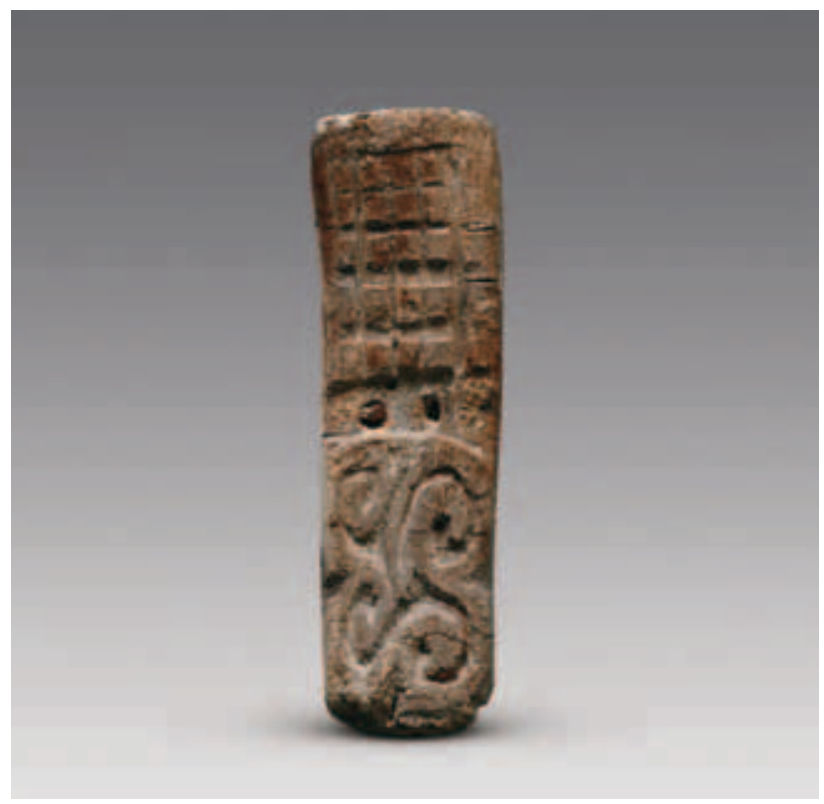

Figure 43 Engraved wooden plaque (Chuvthag 2014M4:21).

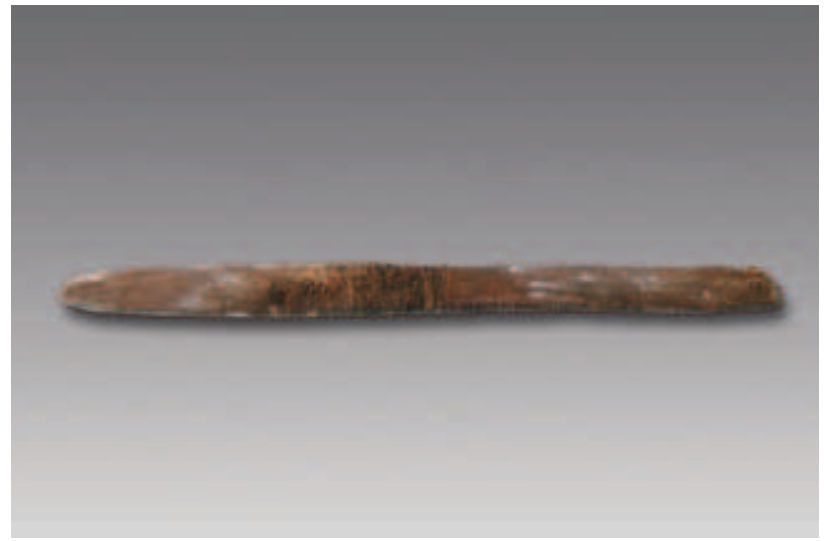

Figure 44 Wooden weaving tool (Chuvthag 2014M4:16). 
covered inside a large pottery jar and was maybe meant to be used for stirring food. The object is of round stick shape with a protrusion on one side. It is $32.3 \mathrm{~cm}$ long and has a diameter of $1.3 \mathrm{~cm}$.

One wooden arrowhead (2013M1:27): the object was made of a thin wooden sheet into the body and the rim of the arrowhead, both of uneven shape. The item is $13.3 \mathrm{~cm}$ long, $2.2 \mathrm{~cm}$ wide, and $0.7 \mathrm{~cm}$ thick.

One bamboo bow (2014M3 right:8): the object was found at the entrance of the burial chamber with an iron arrowhead close-by. It was bent into an arch with traces of bowstring on it. It is $93 \mathrm{~cm}$ long, $1 \mathrm{~cm}$ wide and $3.5 \mathrm{~cm}$ thick.

(3) Gold object: only one mask (2013M1:2); the object is oval in shape, with facial contours including eyebrows, eyes, mouth, and nose impressed onto the gold sheet. At the bottom, there is a pointed chin, almond-shaped eyes, a long nose, and a small mouth. The rim is folded toward the back, probably to affix it to another item. The features are all outlined in red color. The mask is $5.5 \mathrm{~cm}$ long, $4.1 \mathrm{~cm}$ wide and $0.01 \mathrm{~cm}$ thick (Figure 45 ).

(4) Bronzes: 21 items (sets). The main types are mirrors, basins, bracelets, ornaments, beads, and strapcrossings.

One mirror (2014M4:10): the object is severely rusted. The mirror face is round, the rectangular handle has two round holes in it. On the back of the mirror there were four rings of concentric circle pattern. The diameter is $5 \mathrm{~cm}$, the handle is $1.2 \mathrm{~cm}$ long and $0.2 \mathrm{~cm}$ thick.

One basin (2013M1:13): the object was made by forging. It has an angular lip, a slightly contracted rim, a slanted body, and a round bottom. On the outside of the basin wall there were soot traces and inside the vessel there were charred food remains. The rim diameter measures $30.8 \mathrm{~cm}$, the bottom diameter is $20 \mathrm{~cm}$, and the object is $13 \mathrm{~cm}$ high (Figure 46).

One set of beads consisting of 277 items: 2014M2:19 is cylindrical in shape and connected to the other beads with brown rope that may have been used to decorate clothing. The bead is $1.2 \mathrm{~cm}$ long, has and outer diameter of $0.9 \mathrm{~cm}$, an inner diameter of $0.4 \mathrm{~cm}$, and the interval between beads is $2 \mathrm{~cm}$.

Two sets of strung bronze beads: 2014M4:22 consists of 47 bronze beads of different types strung up and with small bronze bells at the end. The beads were in the shapes of round barrel, cylindrical, square column, etc., and have grooves and incised cross-shaped pattern. The beads measure $1-4 \mathrm{~cm}$ in length, with an outer diameter of $0.8 \mathrm{~cm}$, an inner diameter of $0.4 \mathrm{~cm}$, and the whole ornament is $20 \mathrm{~cm}$ long (Figure 47).

Two sets of ornaments, 67 items in total. 2014M2:18 is a set of 42 elements. They are hourglass-shaped rectangular or 8-shaped thin pieces with raised and sunken circle patterns on both sides and a reddish-brown stripe along the waisted middle. They were found on clothing with pieces of textile remains attached to their backs, so they were likely sewn onto clothes, maybe as buttons. They are $2-3 \mathrm{~cm}$ long, $1.5-1.8 \mathrm{~cm}$ wide and $0.2 \mathrm{~cm}$ thick (Figure 48 ).

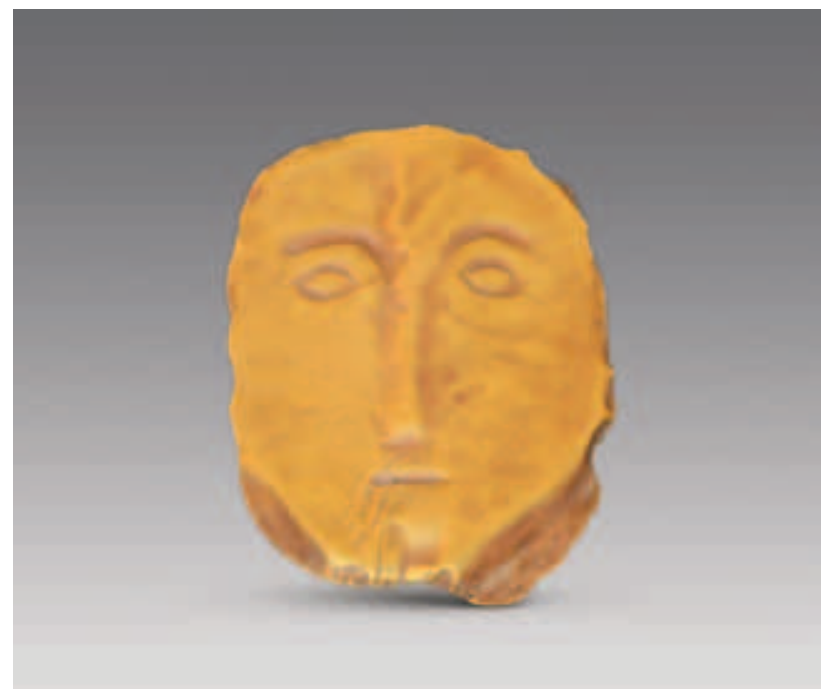

Figure 45 Gold mask (Chuvthag 2013M1:2).

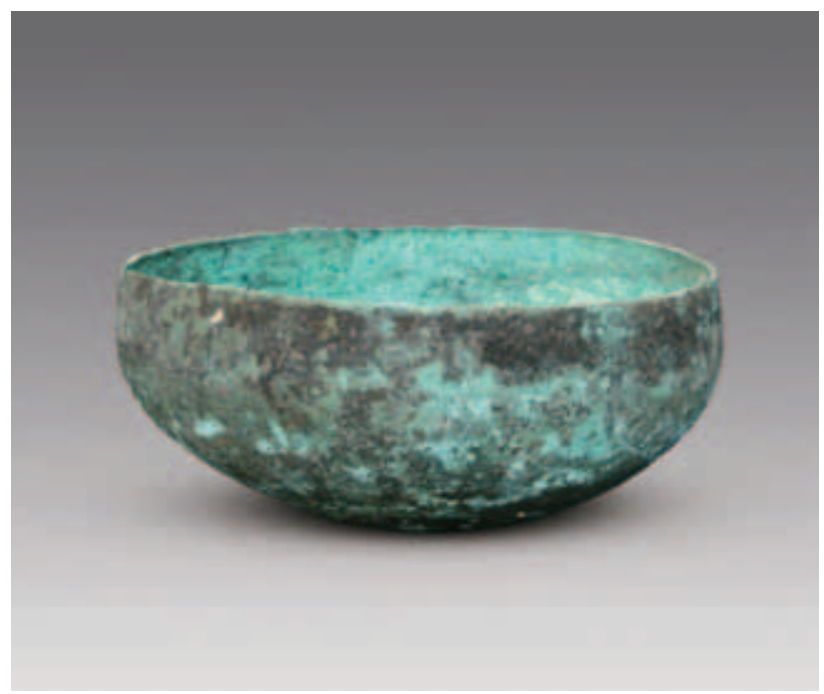

Figure 46 Bronze basin (Chuvthag 2013M1:13).

Four bells: they are conical or spherical in shape with a hollow body, a round ring or hole on the top with thread through, and they were probably used as pendants. 2014M4:23 has a maximum diameter of $1.4 \mathrm{~cm}$ and a height of $2.2 \mathrm{~cm} .2014 \mathrm{M} 4: 24$ has a maximum diameter of $1.7 \mathrm{~cm}$ and a height of $3.5 \mathrm{~cm}$.

One piece of hair ornament (2014M2:23): this object was discovered on the back of the head of the burial occupant. It is rectangular and curving inward to form an arch; on its back there are decors of three S-shaped patterns. It is $4.2 \mathrm{~cm}$ long, $1.5 \mathrm{~cm}$ wide and $0.6 \mathrm{~cm}$ thick.

Five bracelets: 2014M2:24 and 25 form a pair and are rather well preserved; the bracelets are ring-shaped with an opening. They measure $6 \mathrm{~cm}$ in diameter, $1.5 \mathrm{~cm}$ in width, and $0.1 \mathrm{~cm}$ in height (Figure 49). 2014M2:26 consists of a closed circular form with spiral pattern on it. It measures $7.2 \mathrm{~cm}$ in diameter, $1.3 \mathrm{~cm}$ in width and $0.2 \mathrm{~cm}$ in thickness. 


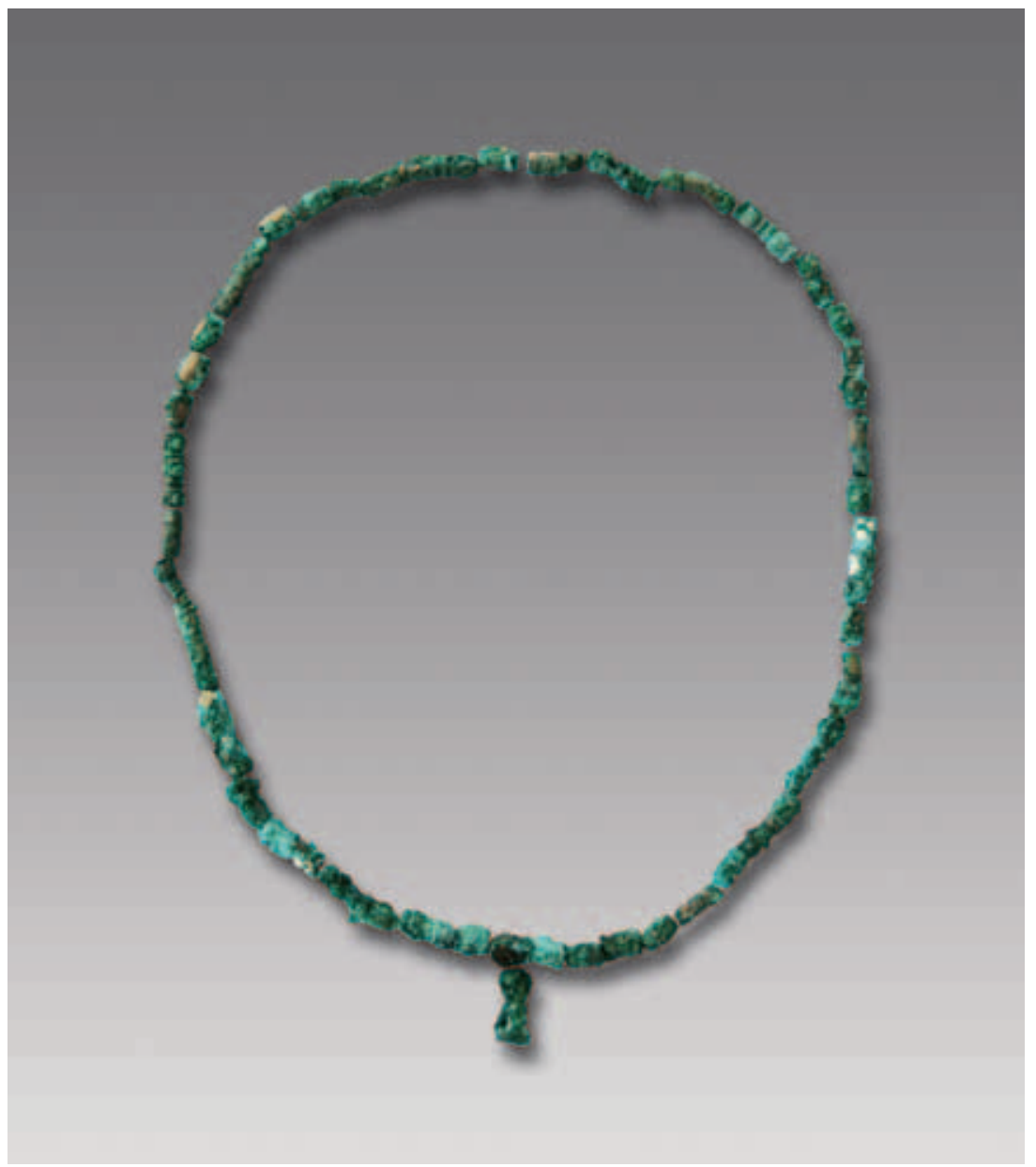

Figure 47 Strung bronze beads (Chuvthag 2014M4:22).

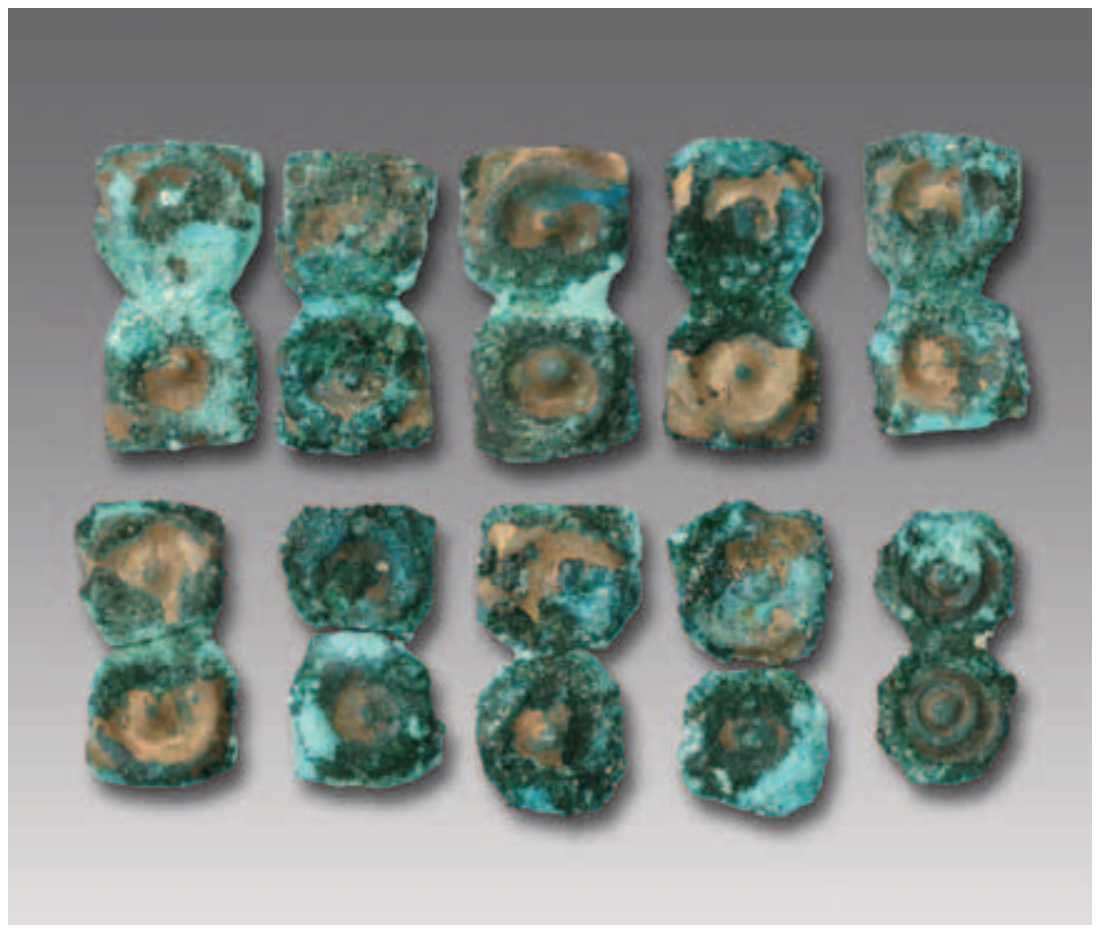

Figure 48 Bronze ornaments (Chuvthag 2014M2:18).
One button (2014M2:27): the button is a flat disk with a round ring on the back. It is decorated with four incised arcs building a square in whose middle there is a diamond shape. The diameter is $2.3 \mathrm{~cm}$, the object is $1.2 \mathrm{~cm}$ high and $0.3 \mathrm{~cm}$ thick.

Three strap-crossings: these items were found by a horse skull and had probably been used together with the horse bit. They are in the shape of round button with a slightly convex top and four oval rings on the back for securing it to the reins. 2014M5:8 measures $3.5 \mathrm{~cm}$ in diameter and is $1.6 \mathrm{~cm}$ high.

(5) Iron objects: 41 objects, mostly plate, awls, cutters, horse bits, belt buckles and arrowheads.

One plate (2013M1:22): the object has a round lip, wide opening, slanted belly, and flat bottom, with a rim diameter of $31 \mathrm{~cm}$, a bottom diameter of $22 \mathrm{~cm}$, and a height of $3.5 \mathrm{~cm}$ (Figure 50).

Six wooden-hilted daggers: they were found next to animal sacrifices. They were severely rusted; the blade is rod-shaped or spearhead-shaped, and inserted into the wooden hilt which has a round cross-section, and secured by silk thread. 2014M3 right:21 is $20 \mathrm{~cm}$ long and $0.6-2.5 \mathrm{~cm}$ wide (Figure 51). 2013M1:25 has a triangular blade, a long hilt, and a clear ridge on the back. It is $22.5 \mathrm{~cm}$ long, has a maximum width of $2.9 \mathrm{~cm}$ and is $1.9 \mathrm{~cm}$ thick.

One cutter (2014M5:15): the blade has a curved cutting edge, rod-shaped tang, between blade and tang is a protruding hand guard, and originally there should have been a wooden hilt around the tang. The item is $16.2 \mathrm{~cm}$ long, measures $2.7 \mathrm{~cm}$ at its widest point, and it is $1.8 \mathrm{~cm}$ thick.

Three hook-shaped objects: the body is straight and the end curved with fragments of a wooden handle, but the function is unclear. 2014 M5:14 is $17.1 \mathrm{~cm} \mathrm{long}, 2.1 \mathrm{~cm}$ wide, and $0.4 \mathrm{~cm}$ thick.

Four horse bits: they consist of two parts linked through a ring in between. 2014M5:13 was found in the mouth of a horse head buried with the burial occupant. It is $22 \mathrm{~cm}$ long and the diameter of the ring measures $3 \mathrm{~cm}$. 
One belt buckle (2014M3 right:22): this item was discovered on horse bones and is severely rusted. It is of circular shape with a tongue in the middle. The diameter is $4.5 \mathrm{~cm}$ and the thickness is $0.7 \mathrm{~cm}$.

16 arrowheads: they are double-winged and have a tang. 2014M3 right:23 (collectively numbered) are 7.5$10 \mathrm{~cm}$ long (Figure 52).

One spoon (2014M5:16): the object is severely corroded. The spoon head is oval and the handle is rod-shaped. The object is $18 \mathrm{~cm}$ long, the spoon head measures $3 \mathrm{~cm}$ in width, and the handle measures $0.6 \mathrm{~cm}$ in cross-section.

Two hairpin-shaped objects: one end of these hairpins consists of two circles curving in opposite directions, and the body is stick-shaped. The pins are incised with spiral pattern. 2014M3 left:10 is $25.1 \mathrm{~cm}$ long with a diameter of $0.5 \mathrm{~cm}$.

Six bracelets: 2014M2:28 was found around the wrist of the burial occupant and was heavily rusted. The bracelet is in the shape of a ring with an opening. The overall diameter is $6.7 \mathrm{~cm}$, the item is $1.5 \mathrm{~cm}$ wide and $0.3 \mathrm{~cm}$ thick.

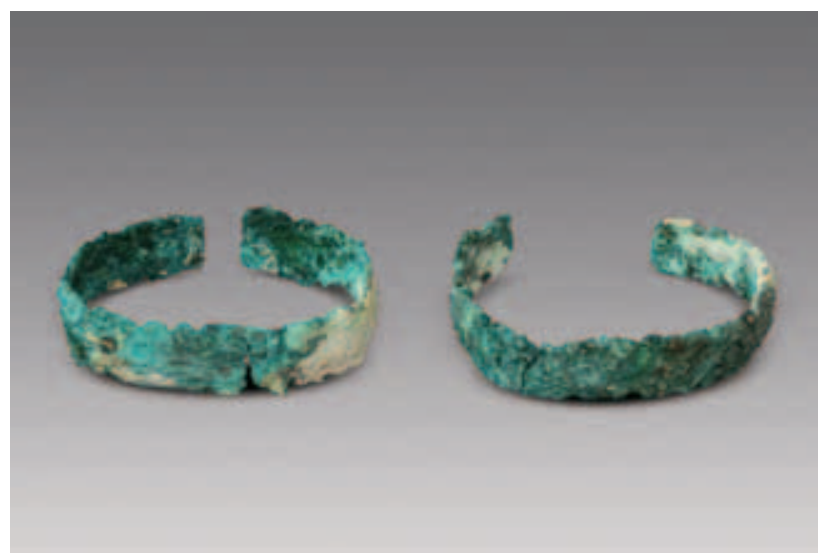

Figure 49 Bronze bracelets (Chuvthag 2014M2:24 and 25).

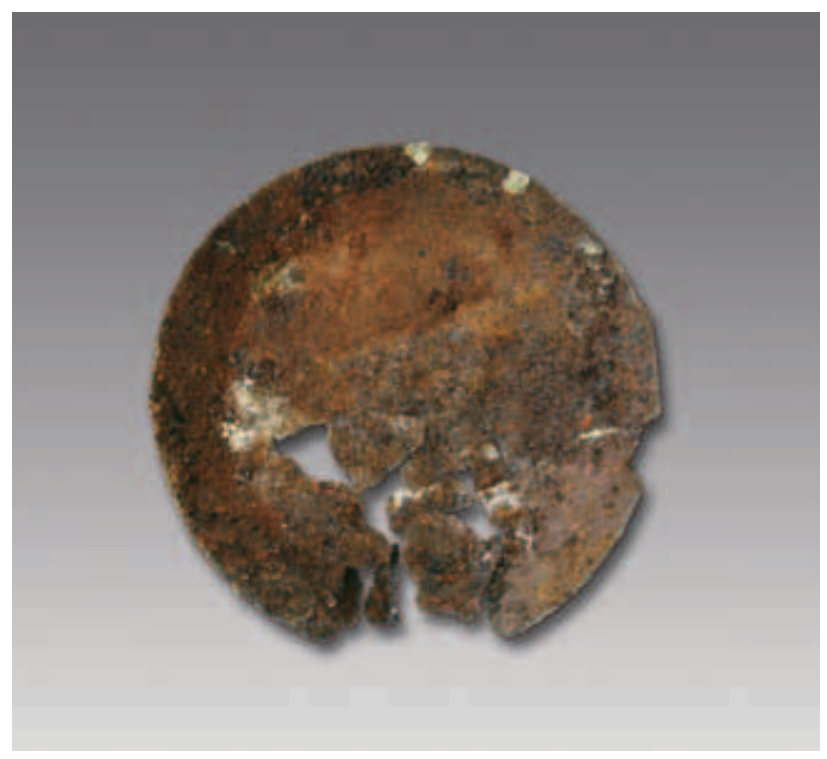

Figure 50 Iron plate (Chuvthag 2013M1:22).
(6) Other objects including beads of various materials, shell ornaments, and basketry items.

Two etched agate beads: 2014M4:15 was found on the head of the burial occupant together with small braids, so it should be an ornament. The bead is olive shaped and flattened on two sides. In the middle-perforation there were some remains of the string on which the bead had been strung. On the surface, there was dark brown and milk white decors building two rows of white triangle pattern with brown wave pattern in between. On both ends about $2.5 \mathrm{~mm}$ of brown band remained. On the middle part, the deep brown color stained the material deeply on one side but not on the other; there the original color shines through. The item is $2.85 \mathrm{~cm}$ long, with a maximum diameter of $0.9 \mathrm{~cm}$ and a diameter of $0.5 \mathrm{~cm}$ on both ends, while the hole measures $0.2 \mathrm{~cm}$ in diameter (Figure 53). 2013M1:3 has an oval-shaped body, and there are remainders of silk thread inside the hole. The body bears patterns in black and white color, white in the center and black on the outside, the pattern being somewhat unclear. The outer

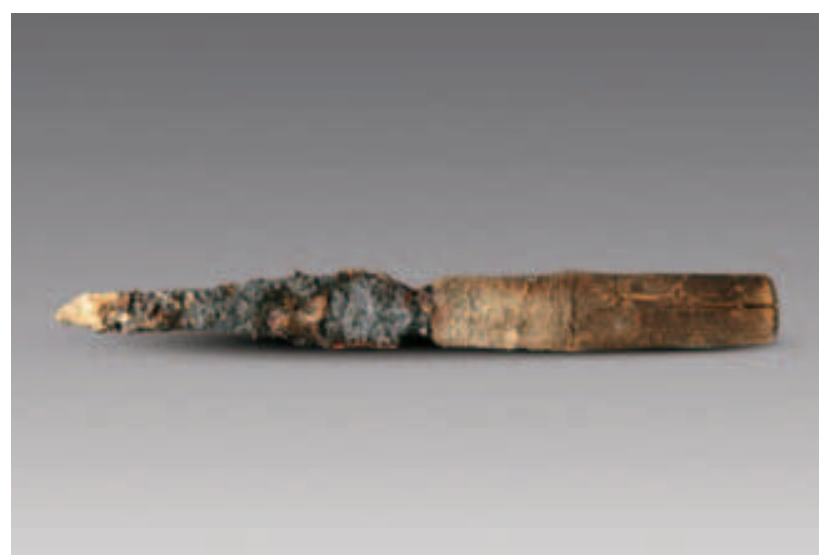

Figure 51 Wooden-hilted iron dagger (Chuvthag 2014M3 right:21).

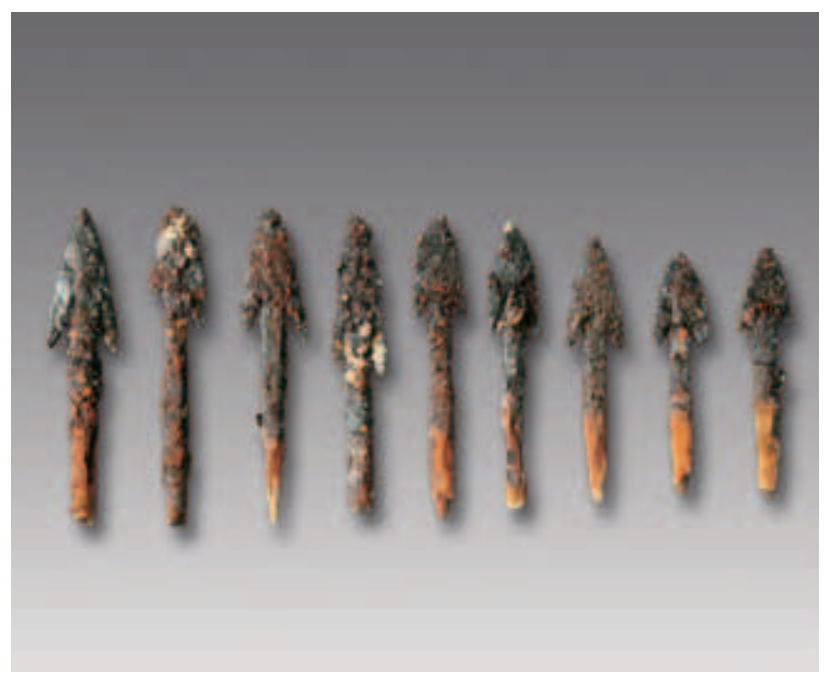

Figure 52 Iron arrowheads (Chuvthag 2014M3 right:23-1 to 23-9). 
diameter is $2.9 \mathrm{~cm}$ and the bead is $1.3 \mathrm{~cm}$ thick while the hole diameter measures $0.2 \mathrm{~cm}$.

37 agate beads: $2014 \mathrm{M} 2$ contained 36 agate beads (2014M2:30) that are all perforated and belonged to the same chain of dark red beads. 18 of them were long and tubular in shape, with a length of $1-3.6 \mathrm{~cm}$ and a diameter of $0.6 \mathrm{~cm} .15$ are short and round with a length of $0.3 \mathrm{~cm}$ and an outer diameter of $0.6 \mathrm{~cm}$. One bead is thick and cylindrical with a length of $0.8 \mathrm{~cm}$ and an outer diameter of $1.5 \mathrm{~cm}$. Two beads are round with a diameter of $0.6-0.8 \mathrm{~cm}$ (Figure 54).

756 glass beads: they were found in 2014M2 (45 beads) and 2014M3 (711 beads) on and around the bodies. They are blue or dark blue and of translucent quality. The outer diameter is $0.2 \mathrm{~cm}$ and the inner diameter is $0.1 \mathrm{~cm}$.

Eight faience beads were unearthed from 2014M2 and probably belonged to the same chain. They are light green in color, tubular in shape, and inside there are remainders of a string they were strung upon. They are $0.65 \mathrm{~cm}$ long with an outer diameter of $0.3 \mathrm{~cm}$ and an inner diameter of $0.2 \mathrm{~cm}$.

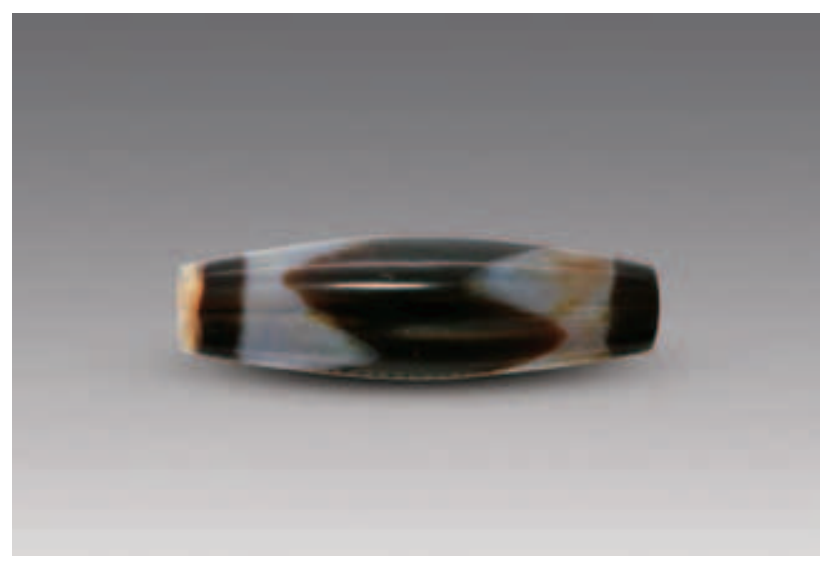

Figure 53 Etched agate bead (Chuvthag 2014M4:15).

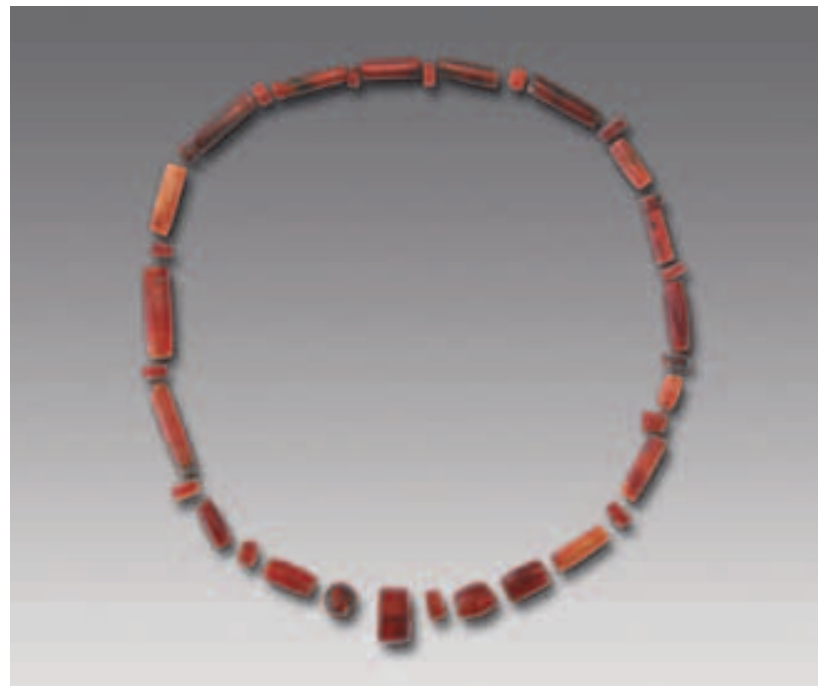

Figure 54 Agate bead necklace (Chuvthag 2014M2:30).
Two shell ornaments: they are white, round, and concave on the inner side. 2013M1:26 is perforated in the middle, has yellow spots on the surface and enamel on the inside. The outer diameter is $4.8 \mathrm{~cm}$, it is $0.9 \mathrm{~cm}$ thick, and the hole diameter is $0.3 \mathrm{~cm} .2014 \mathrm{M} 3$ left: 12 has yellowishbrown spots on the surface and a hole on the side. The outer diameter is $2.6 \mathrm{~cm}$, it is $0.2 \mathrm{~cm}$ thick, and the hole diameter measures $0.2 \mathrm{~cm}$.

Four beads made of job's tear seeds: 2014M3 left:13 likely belonged to a chain. It is of deep brown color, teardrop-shaped, has a perforation, and around this perforation there are scuff marks. The length is $0.85 \mathrm{~cm}$, the outer diameter is $0.55 \mathrm{~cm}$, and the hole diameter is $0.1 \mathrm{~cm}$ (Figure 55).

There are 38 basketry items that were unearthed from inside and outside coffins or from recesses in burial chambers. Most of them are badly decomposed but a few are well preserved. They were made from woven reed. They are round with a wide opening and a small bottom, and they were filled with grass or blackened food remains. 2014M3 right:30 has a rim diameter of $17.5 \mathrm{~cm}$, a bottom diameter of $8.5 \mathrm{~cm}$, and a height of $9.7 \mathrm{~cm}$ (Figure 56).

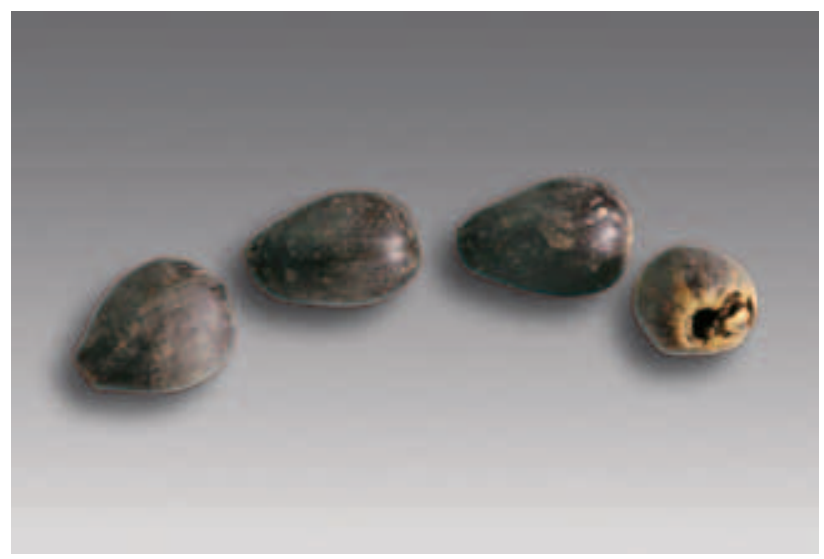

Figure 55 Beads made of job's tear seeds (Chuvthag 2014M3 left:13).

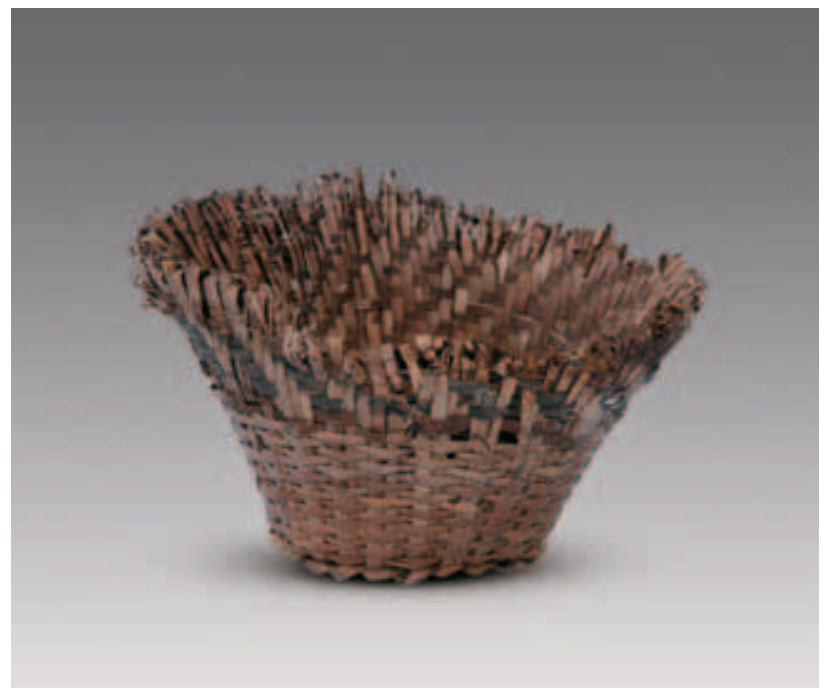

Figure 56 Basketry utensil (Chuvthag 2014M3 right:30). 


\section{Conclusion}

Gurugyam is the largest cemetery with the biggest concentration of burials found so far in the Ngari Prefecture, Tibet, and it is closely connected with Gar City Site (i.e., the legendary capital of Khyunglung Dngul mkhar). Chuvthag is the first cave burial cemetery excavated in Ngari; it has completely preserved graves, a large number of grave goods, finds covering many aspects of past society and daily life, thus contributing substantially to our understanding of pre-Tubo period history and culture. The discoveries and research conducted at these two sites are very important for establishing the cultural sequence of the archaeology of western Tibet, and they represent a major step in research on early Tibetan culture.

The two cemeteries are rather close to each other, they have many elements in common, and they are close in date as well, which is very important for comparing the two cemeteries and establishing an archaeological chronological sequence for western Tibet. According to radiocarbon data taken at both sites, Gurugyam Cemetery and Chuvthag Zone I date to the same period, around 2000-1800 BP. Chuvthag Zone II furnished two radiocarbon dates, the one from M3 being 2250 25 BP, M5 dating to $2150 \pm 25 \mathrm{BP}$, so relatively early.

The graves in both cemeteries were aligned in an orderly fashion, all pointing in the same direction and thus obviously following some form of planning; they may thus have been clan cemeteries. The graves of Chuvthag Zones I and II share the same structure, all of them being earthen cave burials with vertical passage; this type of graves is by no means unique in western Tibet, but there are reports of similar cave burials from Phiyang Dungkar Cemetery (Chinese Institute of Tibetology 2001) and Mkhar phug Cemetery (Huo 2005) in Zanda County. Inside the burial chamber there is some partitioning by function; in all four walls there are all kinds of recesses holding household items packed densely and thus making use of the limited space. This grave structure may imitate the cave dwellings that people lived in at the time, and they are indeed very similar in structure to the cave sites common in this region. It is impossible at present to assess which of these dwellings date to the pre-Tubo period, but it is clear that a few hundred years around the Common Era people were already able to construct this kind of dwellings. The discovery of these burials thus provides us with a glimpse of the structure and use of pre-Tubo dwellings.

The earthen pit burials found at Gurugyam are very different from the cave burials of Chuvthag; they consist of large rectangular pits that were lined with stones to build a grave chamber, a type of structure that is extremely rare in western Tibet and its surroundings. The difference in structure between the two cemeteries is caused by differences in natural environment and cultural affiliation that needs to be analyzed further.

The well-preserved pottery wares unearthed from both cemeteries provide important material for archaeo- logical research on western Tibet during the pre-Tubo period. There are some similarities in pottery form, such as the spouted jars unearthed from Gurugyam that have many counterparts at Chuvthag Cemetery, but overall pottery wares are rare at Gurugyam, they are rather small and coarse. Chuvthag Zones I and II have a rather homogenous pottery assemblage including spouted jars and handled round-bottomed jars which are particularly common, however, there are also clear differences. In Zone II painted wares and double-handled jars are rather popular; in Zone I no painted pottery has been found, but single-handled jars and vessels with trumpet-shaped ring foot have been found in greater number. The pottery assemblages in Zone II are more uniform reflecting a regulated burial custom, their shape and position reflecting clear differences in function. Overall, the quality of these pottery wares is rather high, especially in case of the large jars and the small painted wares with incised decor, they are regular in form and quality, reflecting a developed local pottery tradition that is not very common in western Tibet.

The pottery wares unearthed from Chuvthag Cemetery are closely connected with those from surrounding areas such as Phiyang Dungkar Cemetery in Zandar County and Mkhar phug Cemetery in western Tibet as well as Malari Cemetery in northern India (Bhatt et al. 2008-09) and Leh Cemetery in Ladakh (Francke 1914). Chuvthag shares quite a number of pottery forms and assemblages with these cemeteries, showing that western Tibet was culturally rather unified during the pre-Tubo period.

Other objects unearthed from these burials reflect cultural interaction between the upper Langqên Zangbo River region and surrounding areas, providing us with important first-hand material on the origin and distribution of these objects as well as exchange and trade throughout western Tibet. Besides the large amount of silk discovered previously, this excavation revealed gold masks, Eurasian steppe-style handled bronze mirror, wooden-hilted iron dagger, engraved wooden plaques, wooden combs, and basketry items that that have very close ties to Xinjiang. The large number of hourglass-shaped bronze ornaments, bronze bells, and handled mirrors are similar to objects excavated from Leh Cemetery in Ladakh (Francke 1914), providing a connection for this long-distance interaction which shows that the contact between the upper Langqên Zangbo River region and Xinjiang/Central Asia went through Ladakh. The iron swords with I-shaped hand guard were probably influenced by the Central Plains; bronze basin, bronze mirror, and wooden-hilted dagger are largely identical with objects found in Nepal (Aldenderfer 2013). Some ornaments such as the etched agate beads, glass beads, job's tears seed beads, and shell ornaments are closely connected with finds from Xinjiang, the South Asian subcontinent, and the Indian Ocean area. These new findings show that as early as in the early Iron Age, western Tibet was already closely connected with other cultural centers in its surroundings.

The excavations conducted at these two cemeteries 
also contribute to the research on early Bôn burial rituals. The incomplete human bones found in collective burials and the use of gold masks suggest that the bodies were ritually altered before being buried (Tong 2015); the sacrificial remains inside the burial chambers reflect the emergence of human sacrifice; there is a wide variety and large number of sacrificed animal remains; additionally, various animals were very popularly depicted. These cultural particularities are mentioned in historic literature discussing the "Da Yangtong" Kingdom; in the burials of the same time discovered in the western part of the foothills of the Himalayas similar phenomena are very common, indicating a strong connection with early Bôn funerary customs.

Inside some of the burial chambers large amounts of barley seeds as well as bones of cattle, sheep/goat, and horse interred as animal sacrifices have been found, reflecting that people in the Langqên Zangbo River valley had a way of life that combined pastoral activities with agriculture. The two burials are dated between the 3rd to 2nd centuries BCE and the 2 nd and 3 rd centuries CE, i.e., the period of rule of the Zhangzhong Kingdom. The excavation results show that the Langqên Zangbo River valley was the core region of the Zhangzhong Kingdom as well as the population center of the whole Ngari region and a center of economic and cultural interaction. Many aspects of the culture distributed throughout the western part of the Himalayas are rather unified, but there are also many local particularities.

\section{References}

Aldenderfer, Mark. 2013. Variation in mortuary practice on the early Tibetan plateau and the high Himalayas. Journal of the International Association for Bon Research 1 (Inaugural issue):293-318.
Bhatt, R. C., Kenneth L. Kvamme et al. 2008-2009. Archaeological and geophysical investigations of high mountain cave burials in Uttarakhand Himalaya, India. Indo-kōkokenkyū インド考古研究 (Studies in South Asian art and archaeology) 30:1-16.

Chinese Institute of Tibetology, Sichuan University 四川大学 中国藏学研究所 et al. 2001 . 西藏札达县皮央. 东嘎遗 址古墓群试掘简报 (Trial excavation of ancient tombs on the Phiyang Dungkar Site in Zanda County, Tibet). Kaogu 考古 (Archaeology) 6:14-31.

Francke, August H. 1914. Antiquities of Indian Tibet (Pt. I). Calcutta: Superintendent Government Printing. pp. 68-84.

Huo, Wei 霍巍. 2005. 西藏西部早期文明的考古学探索 (A study on the archaeology of the early civilization of western Tibet). Xizang yanjiu 西藏研究 (Tibetan Studies) 1:43-50.

Institute of Archaeology, CASS and Cultural Relics Conservation Institute of Tibet Autonomous Region. 2014. 西藏阿 里地区噶尔县故如甲木墓地 2012 年发掘报告 (The excavation of the Gurugyam Cemetery in Gar County, Ngari Prefecture, Tibet Autonomous Region in 2012). Kaogu xuebao 考古学报 (Acta archaeologica Sinica) 4:563-87.

Tong, Tao 全涛 and Li, Linhui 李林辉. 2015. 欧亚视野内 的喜马拉雅黄金面具 (The Himalayan gold masks seen in the Eurasian perspective). Kaogu 2:92-102. For English version, see this issue: $85-90$.

\section{Postscript}

The original report published in Kaogu 考古 (Archaeology) 2015.7:29-50 was written by Tao Tong 全涛, Linhui Li 李林辉, Trinley Tsering 赤列次仁 and Ya Yao 姚娅. The photographs were taken by Tao Tong and Cuncun Wang 王存存. This report is translated into English by Anke Hein 安可. 